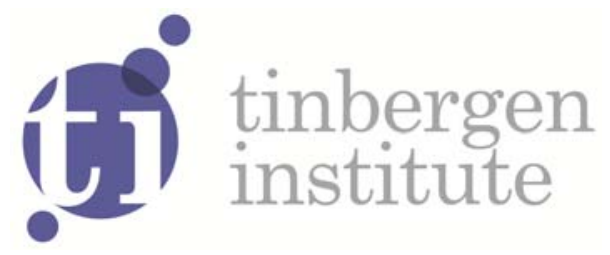

\title{
Auctioning and Selling Positions: A Non-cooperative Approach to Queuing Conflicts
}

René van den Brink'

Youngsub Chun ${ }^{2}$

Yuan Ju

\footnotetext{
1 Faculty of Economics and Business Administration, VU University Amsterdam, and Tinbergen Institute;

2 Department of Economics, Seoul National University, Korea;

3 Department of Economics and Related Studies, University of York, York, United Kingdom.
} 
Tinbergen Institute is the graduate school and research institute in economics of Erasmus University Rotterdam, the University of Amsterdam and VU University Amsterdam.

More TI discussion papers can be downloaded at http://www.tinbergen.nl

Tinbergen Institute has two locations:

Tinbergen Institute Amsterdam

Gustav Mahlerplein 117

1082 MS Amsterdam

The Netherlands

Tel.: +31(0)205251600

Tinbergen Institute Rotterdam

Burg. Oudlaan 50

3062 PA Rotterdam

The Netherlands

Tel.: +31(0)10 4088900

Fax: $+31(0) 104089031$

Duisenberg school of finance is a collaboration of the Dutch financial sector and universities, with the ambition to support innovative research and offer top quality academic education in core areas of finance.

DSF research papers can be downloaded at: http://www.dsf.nl/

Duisenberg school of finance

Gustav Mahlerplein 117

1082 MS Amsterdam

The Netherlands

Tel.: +31(0)20 5258579 


\title{
Auctioning and Selling Positions: a non-cooperative approach to queueing conflicts*
}

\author{
René van den Brink ${ }^{\dagger}$ Youngsub Chun ${ }^{\ddagger}$ Yuan $\mathrm{Ju}^{\S}$
}

January 13, 2014

*Part of the research was conducted when Yuan Ju visited the Department of Economics at Seoul National University, whose hospitality is kindly acknowledged. Chun's work was supported by the National Research Foundation of Korea Grant funded by the Korean Government (NRF2013S1A3A2055391).

${ }^{\dagger}$ Department of Econometrics and Tinbergen Institute, VU University, De Boelelaan 1105, 1081 HV, Amsterdam, The Netherlands. E-mail: jrbrink@feweb.vu.nl.

$\ddagger$ Department of Economics, Seoul National University, Seoul 151-746, Korea. E-mail: ychun@snu.ac.kr.

${ }^{\S}$ Department of Economics and Related Studies, University of York, Heslington, York, YO10 5DD, UK. E-mail: yuan.ju@york.ac.uk. 


\begin{abstract}
Complementary to the axiomatic and mechanism design studies on queueing problems, this paper proposes a strategic bargaining approach to resolve queueing conflicts. Given a situation where players with different waiting costs have to form a queue in order to be served, they firstly compete with each other for a specific position in the queue. Then, the winner can decide to take up the position or sell it to the others. In the former case, the rest of the players will proceed to compete for the remaining positions in the same manner; whereas for the latter case the seller can propose a queue with corresponding payments to the others which can be accepted or rejected. Depending on which position players are going to compete for, the subgame perfect equilibrium outcome of the corresponding mechanism coincides with one of the two best known rules for queueing problems, the maximal and the minimal transfer rules, while an efficient queue is always formed in equilibrium. The analysis discovers a striking relationship between pessimism and optimism in this type of decision making.
\end{abstract}

Keywords: Queueing problem, minimal transfer rule, maximal transfer rule, Shapley value, bidding mechanism, implementation.

JEL code: C71; C72; D60 


\section{Introduction}

Consider a group of agents who want to be severed in a facility. The facility can handle only one agent at a time and agents differ in their unit waiting cost. The queueing problem is concerned with finding the order to serve the agents and the corresponding monetary transfers. This queueing problem has been well studied in the literature from two perspectives: the normative perspective (Maniquet, 2003; Chun, 2006a, b, 2011; Misha and Rangarajan, 2007; Moulin, 2007) and the mechanism design (incentive) perspective (Dolan, 1978; Suijs, 1996; Mitra, 2001, 2002). This paper aims to investigate the problem along an alternative angle, that is, we adopt a strategic approach to build up a natural and intuitive bargaining protocol such that players can negotiate among themselves to resolve the queueing conflicts. Exploring this bargaining approach for queueing problems is not only important in its own right as providing a new toolbox and contributing to an open area of the problem, but has more significant implications: First, it helps understand the strategic features of the allocation rules and makes a fresh review of their plausibility. Next, we can better compare different rules and well associate axiomatic properties with individuals' rational behavior. Furthermore, new insights on fundamental and methodological issues can be developed.

Two well-known rules for the queueing problem were introduced by applying solutions developed for TU (transferable utility) games. Maniquet (2003) introduced the minimal transfer rule, which corresponds to the Shapley value of TU games, when the worth of a coalition is defined to be the minimum waiting cost incurred by its members under the optimistic assumption that they are served before non-coalitional members. On the other hand, Chun (2006a) introduced the maximal transfer rule, which also corresponds to the Shapley value, when the worth of a coalition is defined to be the minimum waiting cost incurred by its members under the pessimistic assumption that they are served after non-coalitional members. Given the connection between the Shapley value for TU games and the minimal and the maximal transfer rules for queueing problems, various bargaining protocols implementing the Shapley value in the literature (Gul, 1989; Hart and Mas-Colell, 1996; Pérez-Castrillo and Wettstein, 2001; Ju and Wettstein, 2009) offer a venue enabling us to construct non-cooperative mechanisms to implement rules for queueing problems.

However, this task is not straightforward, especially when considering that the potential mechanism needs to match the underlying context of the queueing problem. Specifically, one reason is that unlike the TU game, every player's stand-alone value is not well defined in the queueing context. It is conventional in the implementation literature on TU games that when the proposal of a player is rejected in multilateral bargaining she will be left with her stand-alone value, which is not affected by the other players' coalitional behavior and does not affect the other players' payoffs. However, for queueing games, a player's utility 
will necessarily be affected by how the others queue, and where to position this player will also affect the utility of other players. Take for example the queueing game of Maniquet (2003) which is defined in the optimistic perspective. In this game a player's stand-alone value is defined by having this player be served first before anyone else. However, it is impossible to make every player be served first simultaneously in order to apply this standalone value in any bargaining protocol that could be associated with queuing problems. Moreover, if we directly follow the protocol implementing the Shapley value, it would imply a rejected player to be served first in the queue, which not only looks a bit absurd, but also actually fails to implement the minimal transfer rule. Hence, despite their connection, the bargaining protocol for the Shapley value cannot be applied directly to the optimistic game of Maniquet (2003).

The other challenge comes from the incentive design of a proper mechanism for players to form an efficient queue while accepting to make transfers as directed by the minimal transfer rule. One cannot simply assume conditions like super-additivity or zeromonotonicity for queueing problems. Given the specific context, it should be endogenously designed into the mechanism such that players find themselves being better off by building up the efficient queue. A similar remark can be made for the maximal transfer rule and Chun (2006a)'s game in the pessimistic perspective.

In this paper, we construct a non-cooperative mechanism that naturally fits into the context of queueing problems and retains the main feature for TU games, but well overcomes the challenges mentioned above. Players can resolve queueing conflicts by themselves in a decentralized way and guarantee an efficient queue to be formed in equilibrium. By keeping the basic construct and only adjusting a certain detail of the bargaining protocols, we show that the variations can lead to alternative allocation rules for queueing problems. This provides a common platform to study and compare solution concepts for queueing problems and may further help investigate new rules. As we will see, the current study discovers a striking relationship between pessimism and optimism as well as individual and collective behavior. In addition, we offer a strategic foundation for an average transfer rule that takes the average of the maximal and the minimal transfer rules, which can be viewed as a reasonable compromise between the pessimistic and the optimistic perspectives.

The paper is organized as follows. Section 2 provides the preliminaries on queueing problems, including the two rules of queueing problems that we are going to implement. In Section 3 we construct two non-cooperative mechanisms and show that each mechanism has a unique subgame perfect equilibrium outcome which is the allocation prescribed by the maximal transfer rule and, respectively, the minimal transfer rule. We also provide a mechanism to implement the average of the maximal and the minimal transfer rules. In Section 4 we offer a robustness study of the results, which shows that to a great extent the 
ordering of rejected players does not affect the equilibrium outcome.

\section{The queueing problem and two transfer rules}

Let $\mathbb{N}_{++}$be the set of positive natural numbers and $N \subset \mathbb{N}_{++}$be the set of players. A queue $\sigma$ of the set of players $N$ is a bijective function $\sigma: N \rightarrow\{1, \ldots,|N|\}$. The set of all queues of $N$ is denoted by $\Pi(N)$. Each player $i \in N$ takes a position $\sigma_{i}$ in queue $\sigma$ (we write $\sigma_{i}$ instead of $\sigma(i)$ for notational convenience). Given any $\sigma \in \Pi(N)$ and $i \in N$, we denote the set of players proceeding player $i$ by $P_{i}(\sigma)=\left\{j \in N \mid \sigma_{j}<\sigma_{i}\right\}$ and the set of players following $i$ by $F_{i}(\sigma)=\left\{j \in N \mid \sigma_{j}>\sigma_{i}\right\}$. Each player $i \in N$ is characterized by her unit disutility of waiting (unit waiting cost) $\theta_{i} \geq 0$. For any queue $\sigma$, if player $i$ takes the $\sigma_{i}$ th position in this queue, then player $i$ incurs a waiting cost $\left(\sigma_{i}-1\right) \theta_{i}$. Hence, if a player is served first in a queue, there is no time for her to wait to be served and thus, her waiting cost is zero.

A queueing problem is defined by a list $(N, \theta)$ where $\theta=\left(\theta_{i}\right)_{i \in N} \in \mathbb{R}_{+}^{N}$ is the vector of unit waiting costs. Let $\mathbb{Q}^{N}$ be the set of all queueing problems with player set $N$. An allocation for $(N, \theta) \in \mathbb{Q}^{N}$ is a pair $(\sigma, t) \in \Pi(N) \times \mathbb{R}^{N}$, where for each $i \in N, \sigma_{i}$ is the position of $i$ in the queue and $t_{i}$ denotes the monetary transfer to player $i$. Each player $i \in N$ is supposed to have a quasi-linear utility function, and therefore, her utility with respect to the allocation $(\sigma, t)$ is given by $u_{i}(\sigma, t)=-\left(\sigma_{i}-1\right) \theta_{i}+t_{i}$. An allocation $(\sigma, t)$ is feasible if no two players are assigned the same position in the queue $\sigma$ and the sum of all the transfers is not positive. Thus, the set of feasible allocations $\mathcal{F}(N, \theta)$ consists of all $(\sigma, t) \in \Pi(N) \times \mathbb{R}^{N}$ such that for all $i, j \in N$ with $i \neq j$ we have $\sigma_{i} \neq \sigma_{j}$ and $\sum_{i \in N} t_{i} \leq 0$.

Given $(N, \theta) \in \mathbb{Q}^{N}$, an allocation $(\sigma, t) \in \mathcal{F}(N, \theta)$ is efficient if it minimizes the total waiting costs of all players and the transfers are balanced, i.e., all transfers add up to 0. That is, $(\sigma, t) \in \mathcal{F}(N, \theta)$ is efficient if and only if for all $\left(\sigma^{\prime}, t^{\prime}\right) \in \mathcal{F}(N, \theta)$, $\sum_{i \in N}\left(\sigma_{i}-1\right) \theta_{i} \leq \sum_{i \in N}\left(\sigma_{i}^{\prime}-1\right) \theta_{i}$ and $\sum_{i \in N} t_{i}=0$. An efficient queue is a queue that minimizes the total waiting cost of all agents. Note that it has no direct implication on transfers. The set of efficient queues for $(N, \theta) \in \mathbb{Q}^{N}$ is denoted by $\pi(N, \theta)$.

An allocation rule, or a rule, $\varphi$ is a mapping $\varphi: \mathbb{Q}^{N} \rightarrow \mathcal{F}(N, \theta)$, which associates with each problem $(N, \theta) \in \mathbb{Q}^{N}$ a non-empty subset $\varphi(N, \theta) \subset \mathcal{F}(N, \theta)$ of feasible allocations. ${ }^{1}$

There are two prominent rules for the queueing problem: the minimal transfer rule (Maniquet, 2003) and the maximal transfer rule (Chun, 2006a). The minimal transfer rule,

\footnotetext{
${ }^{1}$ When no confusion arises, we write $\varphi(N, \theta)$ instead of $\varphi((N, \theta))$.
} 
$\varphi^{\text {min }}$, is defined by for all $(N, \theta) \in \mathbb{Q}^{N}$,

$$
\varphi^{\min }(N, \theta)=\left\{\left(\sigma^{*}, t^{m i n}\right) \mid \sigma^{*} \in \pi(N, \theta), \text { and } \forall i \in N, t_{i}^{m i n}=\left(\sigma_{i}^{*}-1\right) \frac{\theta_{i}}{2}-\sum_{j \in F_{i}\left(\sigma^{*}\right)} \frac{\theta_{j}}{2}\right\} .
$$

The maximal transfer rule, $\varphi^{\max }$, is defined by for all $(N, \theta) \in \mathbb{Q}^{N}$,

$$
\varphi^{\max }(N, \theta)=\left\{\left(\sigma^{*}, t^{\max }\right) \mid \sigma^{*} \in \pi(N, \theta), \text { and } \forall i \in N, t_{i}^{\max }=\sum_{j \in P_{i}\left(\sigma^{*}\right)} \frac{\theta_{j}}{2}-\left(|N|-\sigma_{i}^{*}\right) \frac{\theta_{i}}{2}\right\} .
$$

Following Maniquet (2003), the queueing problem can be solved by applying solutions of TU (transferable utility) games. To do this, queueing problems should be mapped into TU games. First, we formally describe TU games. Let $N \subset \mathbb{N}_{++}$be the set of players. A set $S \subseteq N$ is a coalition. A TU game is a real-valued function $v$ defined on all coalitions $S \subseteq N$ satisfying $v(\emptyset)=0$. The number $v(S)$ is the worth of $S$. Let $\Gamma^{N}$ be the class of games with player set $N$. A value is a function $\phi$ defined on $\Gamma^{N}$ which associates with every $v \in \Gamma^{N}$ a vector $\phi(v)=\left(\phi_{i}(v)\right)_{i \in N}$.

The Shapley (1953) value, $\phi^{S h}$, assigns to every TU game its expected marginal contribution vector, over all possible coalitions while assuming all the orders of forming into the grand coalition by a one-by-one sequence are equally likely to happen. For all $v \in \Gamma^{N}$ and all $i \in N$,

$$
\phi_{i}^{S h}(v)=\sum_{S \subseteq N \backslash\{i\}} \frac{|S| !(|N|-|S|-1) !}{|N| !}(v(S \cup\{i\})-v(S)) .
$$

Maniquet (2003) introduces a queueing game by taking an optimistic treatment on coalitional waiting costs. For each queueing problem $(N, \theta) \in \mathbb{Q}^{N}$, the optimistic way to construct a queueing game is to assume a coalition $S \subseteq N$ to be served before the rest of the players in $N$. Accordingly, the worth $v^{\min }(S)$ of coalition $S$ is defined as the sum of its members' waiting cost when they arrive before $N \backslash S$ and arrange themselves into an efficient queue. Formally, for all $(N, \theta) \in \mathbb{Q}^{N}$ and all $S \subseteq N$,

$$
v^{\min }(S)=-\sum_{i \in S}\left(\sigma_{i}^{*}-1\right) \theta_{i},
$$

where $\sigma^{*} \in \pi\left(S, \theta_{S}\right)$ and $\theta_{S}=\left(\theta_{i}\right)_{i \in S}$. Note that the right hand side is the same for all $\sigma^{*} \in \pi\left(S, \theta_{S}\right)$. Apparently, $v^{\min }(S)$ is the minimum waiting cost that $S$ can achieve, since all other players $N \backslash S$ are required to be served after $S$. We note that, for any $(N, \theta) \in \mathbb{Q}^{N}$, the resulting payoff to each player by the Shapley value of the associated game $v^{\text {min }}$ is the same as the utility received by the player based on the minimal transfer rule, i.e. $\phi_{i}^{S h}\left(v^{\min }\right)=-\left(\sigma_{i}^{*}-1\right) \frac{\theta_{i}}{2}-\sum_{j \in F_{i}\left(\sigma^{*}\right)} \frac{\theta_{j}}{2}$. 
For all $i \in N$ and all $S \subseteq N \backslash\{i\}$, consider an efficient queue $\sigma^{*}$ for $S \cup\{i\}$, i.e., $\sigma^{*} \in \pi\left(S \cup\{i\}, \theta_{S \cup\{i\}}\right)$, and an efficient queue $\sigma^{* *}$ for $S$, i.e., $\sigma^{* *} \in \pi\left(S, \theta_{S}\right)$. Then, the marginal contribution of $i \in N$ to $S \subseteq N \backslash\{i\}$ in $v^{\text {min }}$ equals

$$
\begin{aligned}
& v^{\min }(S \cup\{i\})-v^{\min }(S) \\
= & -\sum_{k \in S \cup\{i\}}\left(\sigma_{k}^{*}-1\right) \theta_{k}-\left(-\sum_{j \in S}\left(\sigma_{j}^{* *}-1\right) \theta_{j}\right) \\
= & \sum_{j \in S} \sigma_{j}^{* *} \theta_{j}-\sum_{k \in S \cup\{i\}} \sigma_{k}^{*} \theta_{k}+\theta_{i} \\
= & \left(\sum_{j \in P_{i}\left(\sigma^{*}\right)} \sigma_{j}^{*} \theta_{j}+\sum_{j \in F_{i}\left(\sigma^{*}\right)}\left(\sigma_{j}^{*}-1\right) \theta_{j}\right)-\left(\sum_{j \in P_{i}\left(\sigma^{*}\right)} \sigma_{j}^{*} \theta_{j}+\sigma_{i}^{*} \theta_{i}+\sum_{j \in F_{i}\left(\sigma^{*}\right)} \sigma_{j}^{*} \theta_{j}\right)+\theta_{i} \\
= & \left(1-\sigma_{i}^{*}\right) \theta_{i}-\sum_{j \in F_{i}\left(\sigma^{*}\right)} \theta_{j},
\end{aligned}
$$

where the first equality follows by definition of $v^{\text {min }}$, and the third equality follows from the fact that $\sigma_{j}^{* *}=\sigma_{j}^{*}$ for all $j \in P_{i}\left(\sigma^{*}\right)$ and $\sigma_{j}^{* *}=\sigma_{j}^{*}-1$ for all $j \in F_{i}\left(\sigma^{*}\right)$. That is, with the joining of player $i$ to $S$, construction of the efficient queue requires those who have higher waiting costs than $\theta_{i}$ to be served before $i$, and those who have lower waiting costs than $\theta_{i}$ to be served after $i$. Hence, compared to the total cost within $S, i$ 's marginal contribution (in terms of cost) is $\left(1-\sigma_{i}^{*}\right) \theta_{i}-\sum_{j \in F_{i}\left(\sigma^{*}\right)} \theta_{j}$.

Alternatively, Chun (2006a) constructs a pessimistic counter-part of the queueing game for which the worth $v^{\max }(S)$ of a coalition $S \subseteq N$ is defined as the sum of its members' waiting cost when they arrive behind $N \backslash S$ and then arrange themselves into an efficient queue. Formally, for all $(N, \theta) \in \mathbb{Q}^{N}$ and all $S \subseteq N$,

$$
v^{\max }(S)=-\sum_{i \in S}\left((|N|-|S|)+\sigma_{i}^{*}-1\right) \theta_{i}
$$

where $\sigma^{*} \in \pi\left(S, \theta_{S}\right)$ and $\theta_{S}=\left(\theta_{i}\right)_{i \in S}$. Again, the right hand side is the same for all $\sigma^{*} \in \pi\left(S, \theta_{S}\right)$. Note that, for any $(N, \theta) \in \mathbb{Q}^{N}$, the resulting payoff to each player by the Shapley value of the associated game $v^{\max }$ is the same as the utility received by the player based on the maximal transfer rule, i.e. $\phi_{i}\left(v^{\max }\right)=-(|N|-1) \theta_{i}+\sum_{j \in P_{i}\left(\sigma^{*}\right)} \frac{\theta_{j}}{2}+\left(|N|-\sigma_{i}^{*}\right) \frac{\theta_{i}}{2}$.

For all $i$ and all $S \subseteq N \backslash\{i\}$, consider an efficient queue $\sigma^{*}$ for $S \cup\{i\}$, i.e., $\sigma^{*} \in$ $\pi\left(S \cup\{i\}, \theta_{S \cup\{i\}}\right)$, and an efficient queue $\sigma^{* *}$ for $S$, i.e., $\sigma^{* *} \in \pi\left(S, \theta_{S}\right)$. Then, the marginal contribution of player $i \in N$ to coalition $S \subseteq N \backslash\{i\}$ in $v^{\text {max }}$ equals

$$
\begin{aligned}
& v^{\max }(S \cup\{i\})-v^{\max }(S) \\
= & -\sum_{k \in S \cup\{i\}}\left(|N|-|S|-1+\sigma_{k}^{*}-1\right) \theta_{k}-\left(-\sum_{j \in S}\left(|N|-|S|+\sigma_{j}^{* *}-1\right) \theta_{j}\right)
\end{aligned}
$$




$$
\begin{aligned}
= & \sum_{j \in S}\left(|N|-|S|+\sigma_{j}^{* *}\right) \theta_{j}-\sum_{k \in S \cup\{i\}}\left(|N|-|S|-1+\sigma_{k}^{*}\right) \theta_{k}+\theta_{i} \\
= & \left(\sum_{j \in P_{i}\left(\sigma^{*}\right)}\left(|N|-|S|+\sigma_{j}^{*}\right) \theta_{j}+\sum_{j \in F_{i}\left(\sigma^{*}\right)}\left(|N|-|S|+\sigma_{j}^{*}-1\right) \theta_{j}\right) \\
& -\left(\sum_{j \in P_{i}\left(\sigma^{*}\right)}\left(|N|-|S|-1+\sigma_{j}^{*}\right) \theta_{j}+\left(|N|-|S|-1+\sigma_{i}^{*}\right) \theta_{i}+\sum_{j \in F_{i}\left(\sigma^{*}\right)}\left(|N|-|S|-1+\sigma_{j}^{*}\right) \theta_{j}\right) \\
& +\theta_{i} \\
= & \left(2-|N|+|S|-\sigma_{i}^{*}\right) \theta_{i}+\sum_{j \in P_{i}\left(\sigma^{*}\right)} \theta_{j},
\end{aligned}
$$

where equalities are similarly obtained as in (2.1).

\section{Bargaining games for the queueing problem}

In this section, following a natural and intuitive idea that well fits the context of queueing problems, we construct two related bargaining games that implement the maximal and the minimal transfer rules. These mechanisms provide a strategic perspective to evaluate and compare the two rules. Throughout the paper, players are assumed to be risk neutral and expected utility maximizers.

The first game, called the first-served mechanism, which implements the maximal transfer rule, can be described informally as follows. At stage 1 all players participate in a multi-bidding auction to compete for the first position of a queue. In this auction, each player bids by submitting an $(n-1)$-tuple of numbers (positive or negative), one number for each player (excluding herself). Note that a positive number implies a payment she makes to other player and a negative number means a demand she asks for from other player. The player whose net bid (the difference between the sum of bids made by the player and the sum of bids the other players made to her) is the highest wins the first position while making the payment or receiving the compensation, as per the corresponding bid she makes. At stage 2, the winner has two options. She can either take up the first position by herself or sell it to other players. If she decides to take up the position by herself, then the rest of the players will play the game again from the first stage to bargain over those positions after her. If she decides to sell the position, then this sale cannot be a bilateral one because where to locate the winner after the sale affects other players' positions. Therefore, selling the first position will naturally be an all-party negotiation process. That is, the winner will make an overall proposal that consists of a queue assigning positions to all players and a vector of transfers specifying the amount each player is supposed to pay or receive. Stage 3 is to approve or disapprove the proposal. The proposal is accepted if all the other players 
agree. In case of acceptance the proposal is implemented so that the queue is formed with transfers in effect to all players. In case of rejection the proposer loses any say about the queue but she will retain the first position, ${ }^{2}$ although she will incur no additional transfers, i.e., neither pay to others nor receive any compensation from others (except for the bids made at stage 1). Meanwhile, all players except for the rejected proposer start the new round of negotiation using the same rule.

Now we formally describe the first-served mechanism.

First-served mechanism. If there is only one player, $N=\{i\}$, she simply is served first (also last) and the default queue is trivially efficient while no transfer will be made. So this player's utility will be $u_{i}(\sigma, t)=-\left(\sigma_{i}-1\right) \theta_{i}+t_{i}=-0 \theta_{i}+0=0$, which is independent of $\theta_{i}$. When the player set $N=\{1, \ldots, n\}$ consists of two or more players, the mechanism is defined for any set of (active) players $S \subseteq N$, recursively starting with $S=N$.

Stage 1. Bidding for the first position: Each player $i \in S$ makes $s-1$ (where $s=|S|$ is the cardinality of coalition $S$ ) bids $b_{j}^{i} \in \mathbb{R}$, one to every $j \in S \backslash\{i\}$. For each $i \in S$, define the net bid of player $i$ by $B^{i}=\sum_{j \in S \backslash\{i\}} b_{j}^{i}-\sum_{j \in S \backslash\{i\}} b_{i}^{j}$. Let $i_{s}=\operatorname{argmax}_{i \in S} B^{i}$. In case of a non-unique maximizer we choose with equal probability any of these maximal bidders to be the 'winner.' Once the winner $i_{s}$ has been chosen, player $i_{s}$ pays every player $j \in S \backslash\left\{i_{s}\right\}$ her bid $b_{j}^{i_{s}}$.

Stage 2. Taking up or selling the position: The winner $i_{s}$ decides to either take up the first position by herself or sell it to the others. If taking up the position by herself, player $i_{s}$ will be located before all players of $S \backslash\left\{i_{s}\right\}$ but after $N \backslash S$, i.e., with position $|N|-|S|+1$. Hence, her final utility is $-(|N|-|S|) \theta_{i}-\sum_{j \in S \backslash\left\{i_{s}\right\}} b_{j}^{i_{s}}+\sum_{r=s+1}^{n} b_{i_{s}}^{i_{r}}$, where $(|N|-|S|) \theta_{i}$ is her waiting cost at this position, $b_{j}^{i_{s}}$ is the bid she pays to player $j \in S \backslash\left\{i_{s}\right\}$, and $b_{i_{s}}^{i_{r}}, r \in\{s+1, \ldots, n\}$, are the bids she received from the rejected proposers $i_{r}, r \in\{s+1, \ldots, n\}$. Moreover, stage 3 will not be evoked, but all players in $S$ other than $i_{s}$ proceed again from stage 1 where the set of active players is $S \backslash\left\{i_{s}\right\}$. If the winner $i_{s}$ decides to sell the position, then $i_{s}$ will make a proposal $\left(\sigma(S),\left(t_{j}\right)_{j \in S}\right)$ consisting of a queue $\sigma(S) \in \Pi(S)$ and a vector of transfers $\left(t_{j}\right)_{j \in S} \in \mathbb{R}^{S}$ such that $\sum_{j \in S} t_{j} \leq 0$. (This offer is additional to the bids paid at stage 1.) The game continues to stage 3 .

Stage 3. Approving or disapproving a proposal: The players in $S$ other than $i_{s}$, sequentially, either accept or reject the proposal. If at least one player rejects the

\footnotetext{
${ }^{2}$ Indeed this option makes the choice of taking up the first position at stage 2 strategically redundant. Yet it seems natural and logical for the winner to have the right to take up the position without proceeding to the next stage.
} 
proposal, then it is rejected. Otherwise, it is accepted.

(i) If the proposal is rejected, all players in $S$ other than $i_{s}$ go back to stage 1 where the set of active players is $S \backslash\left\{i_{s}\right\}$. Meanwhile, player $i_{s}$ will simply fall back to her default position: the position in front of the queue of $S \backslash\left\{i_{s}\right\}$ but after $N \backslash S$. Consequently, player $i_{s}$ receives her final utility $-(|N|-|S|) \theta_{i}-\sum_{j \in S \backslash\left\{i_{s}\right\}} b_{j}^{i_{s}}+\sum_{r=s+1}^{n} b_{i_{s}}^{i_{r}}$. Note that this is the same utility she would receive if she decided in stage 2 to take up her position.

(ii) If the proposal is accepted, then we have to distinguish between two cases, $S=N$ and $S \neq N$. If $S=N$, all players agree with the proposer $i_{n}$ on her proposal $\left(\sigma(N),\left(t_{j}\right)_{j \in N}\right)$ and the game ENDS. Each player $j \in N \backslash\left\{i_{n}\right\}$ receives $-\left(\sigma_{j}(N)-\right.$ 1) $\theta_{j}+b_{j}^{i_{n}}+t_{j}$, and player $i_{n}$ receives $-\left(\sigma_{i_{n}}(N)-1\right) \theta_{i_{n}}-\sum_{j \in N \backslash\left\{i_{n}\right\}} b_{j}^{i_{n}}+t_{i_{n}}$, where $t_{i_{n}}=$ $-\sum_{j \in N \backslash\left\{i_{n}\right\}} t_{j}$. If $S \neq N$, all players in $S$ agree with the proposer $i_{s}$ on her proposal $\left(\sigma(S),\left(t_{j}\right)_{j \in S}\right)$, and the game ENDS. Each player $j \in S \backslash\left\{i_{s}\right\}$ receives $-(|N|-|S|+$ $\left.\sigma_{j}(S)-1\right) \theta_{j}+t_{j}$, and with the bids made by $i_{s}$ and all other previously rejected proposers, player $j$ 's final utility is $-\left(|N|-|S|+\sigma_{j}(S)-1\right) \theta_{j}+\sum_{k=s}^{n} b_{j}^{i_{k}}+t_{j}$. Player $i_{s}$ receives $-\left(|N|-|S|+\sigma_{i_{s}}(S)-1\right) \theta_{i_{s}}-\sum_{j \in S \backslash\left\{i_{s}\right\}} b_{j}^{i_{s}}+t_{i_{s}}$, where $t_{i_{s}}=-\sum_{j \in S \backslash\left\{i_{s}\right\}} t_{j}$, and adding the bids $i_{s}$ received from the previously rejected proposers, the final utility of player $i_{s}$ is $-\left(|N|-|S|+\sigma_{i_{s}}(S)-1\right) \theta_{i_{s}}-\sum_{j \in S \backslash\left\{i_{s}\right\}} b_{j}^{i_{s}}+\sum_{k=s+1}^{n} b_{i_{s}}^{i_{k}}+t_{i_{s}}$.

We show that for any queueing problem $(N, \theta) \in \mathbb{Q}^{N}$, the first-served mechanism has a unique subgame perfect equilibrium (SPE) outcome, which coincides with the payoff vector prescribed by the maximal transfer rule. We first present the following lemma.

Lemma 3.1 For all $(N, \theta) \in \mathbb{Q}^{N}$, all $i \in N$, and all $S \subseteq N \backslash\{i\}$,

$$
v^{\max }(S \cup\{i\})-v^{\max }(S) \geq-(|N|-|S|-1) \theta_{i} .
$$

Proof. Let $T \subseteq S$ be the set of players whose unit waiting costs are greater than $\theta_{i}$, and $S \backslash T$ be the set of players whose unit waiting costs are less than or equal to $\theta_{i}$. That is, $\theta_{k} \leq \theta_{i}<\theta_{j}$ for all $j \in T$ and all $k \in S \backslash T$. In an efficient queue $\sigma^{*}$ for $S \cup\{i\}$, player $i$ will be served after players in $T$ but before anyone else in the queue $\sigma^{*}$, so that $\sigma_{i}^{*}=|T|+1$. $^{3}$ Thus,

$$
v^{\max }(S \cup\{i\})-v^{\max }(S)=\left(2-|N|+|S|-\sigma_{i}^{*}\right) \theta_{i}+\sum_{j \in P_{i}\left(\sigma^{*}\right)} \theta_{j}
$$

\footnotetext{
${ }^{3}$ Note that the position of player $i$ may not be $|T|+1$ if there is a player $j \in S$ with $\theta_{j}=\theta_{i}$. Since the choice of an efficient queue has no effect on $v^{\max }(S \cup\{i\})-v^{\max }(S)$, we can take an efficient queue $\sigma^{*}$ with $\sigma_{i}^{*}=|T|+1$.
} 


$$
\begin{aligned}
& =(2-|N|+|S|-(|T|+1)) \theta_{i}+\sum_{j \in T} \theta_{j} \\
& =-(|N|-|S|+|T|-1) \theta_{i}+\sum_{j \in T} \theta_{j} \\
& \geq-(|N|-|S|+|T|-1) \theta_{i}+|T| \theta_{i} \\
& =-(|N|-|S|-1) \theta_{i} .
\end{aligned}
$$

The inequality in Lemma 3.1 (and the proof) is strict if $\theta_{i}<\theta_{j}$ for at least one $j \in S \backslash\{i\} .{ }^{4}$ The implication of this lemma is rather intuitive: it pays for player $i$ to join coalition $S$ and form an efficient queue rather than taking up a position in front of $S$ since the cost for the coalition $S \cup\{i\}$ is not more than the cost of $S$ plus the cost of $i$ when she is served in the position before $S$. The right hand side of the inequality of the lemma is the utility of player $i$ when she takes up the position $\sigma_{i}=|N|-|S|$. Although this result shares a similar feature as the zero-monotonicity property for TU games, the difference lies in the fact that the zero-monotonicity property makes a comparison between the marginal contribution of a player and her stand-alone worth. Note that the stand-alone worth of player $i$ in this game is $-(|N|-1) \theta_{i}$. Moreover, the cost $-(|N|-|S|-1) \theta_{i}$ depends on the size of $S$, while the stand-alone worth $v^{\max }(\{i\})=-(|N|-1) \theta_{i}$ does not.

Theorem 3.2 For any queueing problem $(N, \theta) \in \mathbb{Q}^{N}$, the first-served mechanism has a unique subgame perfect equilibrium (SPE) outcome, which coincides with the payoff vector prescribed by the maximal transfer rule $\varphi^{\max }(N, \theta)$.

Proof. Consider a queueing problem $(N, \theta) \in \mathbb{Q}^{N}$. The proof proceeds by induction on the number of players $n$. The induction assumption is that whenever the mechanism is used by $n$ players with a given vector of unit waiting costs, it implements the maximal transfer rule of this queueing problem. It is easy to see that the theorem holds for $n=1$. We assume that it holds for all $m \leq n-1$ and show that it is satisfied for $n$.

First we show that the maximal transfer allocation is an SPE outcome. We explicitly construct an SPE that yields the maximal transfer allocation as the SPE outcome. Consider the following strategies, which the players would follow in any (sub)game they participate in (we describe it for the whole set of players, $N$, but similar strategies are followed by any player in $S \subseteq N$ that is called upon to play the game, with $S$ replacing $N$ ):

\footnotetext{
${ }^{4}$ In the proof, if $T \neq \emptyset$, then the inequality becomes strict.
} 
At stage 1, each player $i \in N$ announces

$$
\begin{aligned}
b_{j}^{i}= & \left(-\left(\sigma_{j}^{*}-1\right) \theta_{j}+\sum_{k \in P_{j}\left(\sigma^{*}\right)} \frac{\theta_{k}}{2}-\left(|N|-\sigma_{j}^{*}\right) \frac{\theta_{j}}{2}\right) \\
- & \left(-\left(1+\sigma_{j}^{* *}-1\right) \theta_{j}+\sum_{k \in P_{j}\left(\sigma^{* *}\right)} \frac{\theta_{k}}{2}-\left(|N|-1-\sigma_{j}^{* *}\right) \frac{\theta_{j}}{2}\right)
\end{aligned}
$$

where $\sigma^{*} \in \pi(N, \theta)$ and $\sigma^{* *} \in \pi\left(N \backslash\left\{i_{n}\right\}, \theta_{N \backslash\left\{i_{n}\right\}}\right)$ for all $j \in N \backslash\{i\}$.

At stage 2 , the proposer ('winner' of the bidding in stage 1), player $i_{n}$, adopts the option of selling the position instead of taking it up by herself, and makes a proposal $\left(\sigma^{*}, t\right)$ such that $\sigma^{*} \in \pi(N, \theta)$ and for all $j \in N \backslash\left\{i_{n}\right\}$,

$t_{j}=\left(\sigma_{j}^{*}-1\right) \theta_{j}+\left(-\left(1+\sigma_{j}^{* *}-1\right) \theta_{j}+\sum_{k \in P_{j}\left(\sigma^{* *}\right)} \frac{\theta_{k}}{2}-\left(|N|-1-\sigma_{j}^{* *}\right) \frac{\theta_{j}}{2}\right)$, where $\sigma^{* *} \in$ $\pi\left(N \backslash\left\{i_{n}\right\}, \theta_{N \backslash\left\{i_{n}\right\}}\right)$.

At stage 3 , any player $j \in N \backslash\left\{i_{n}\right\}$ accepts any proposal $(\sigma, t)$ such that $\sigma \in \Pi(N)$ and $t_{j} \geq\left(\sigma_{j}-1\right) \theta_{j}+\left(-\left(1+\sigma_{j}^{* *}-1\right) \theta_{j}+\sum_{k \in P_{j}\left(\sigma^{* *}\right)} \frac{\theta_{k}}{2}-\left(|N|-1-\sigma_{j}^{* *}\right) \frac{\theta_{j}}{2}\right)$ where $\sigma^{* *} \in$ $\pi\left(N \backslash\left\{i_{n}\right\}, \theta_{N \backslash\left\{i_{n}\right\}}\right)$, and rejects it otherwise. (Note that the right hand side is the same for any $\sigma^{* *} \in \pi\left(N \backslash\left\{i_{n}\right\}, \theta_{N \backslash\left\{i_{n}\right\}}\right)$.)

To verify that the above strategies indeed constitute an SPE and yield the maximal transfer allocation, we first look at stage 3 . Suppose the proposal of $i_{n}$ is rejected. From the induction hypothesis, we know that for the remaining players in $N \backslash\left\{i_{n}\right\}$, a corresponding efficient queue will be formed in the unique SPE outcome of that subgame and the resulting utility to every player $j \in N \backslash\left\{i_{n}\right\}$ is $-\left(1+\sigma_{j}^{* *}-1\right) \theta_{j}+\sum_{k \in P_{j}\left(\sigma^{* *}\right)} \frac{\theta_{k}}{2}-\left(|N|-1-\sigma_{j}^{* *}\right) \frac{\theta_{j}}{2}$, where $\sigma^{* *} \in \pi\left(N \backslash\left\{i_{n}\right\}, \theta_{N \backslash\left\{i_{n}\right\}}\right)$, which is the reservation utility for $j$ when she considers any proposal made by $i_{n}$. If $j$ accepts the proposal made by $i_{n}$, then she is located at position $\sigma_{j}$ with transfer $t_{j}$ so that she receives the utility $-\left(\sigma_{j}-1\right) \theta_{j}+t_{j}$. Apparently, only when this utility is no less than her reservation utility, it is possible for her to accept the proposal, which gives rise to $t_{j} \geq\left(\sigma_{j}-1\right) \theta_{j}+\left(-\left(1+\sigma_{j}^{* *}-1\right) \theta_{j}+\sum_{k \in P_{j}\left(\sigma^{* *}\right)} \frac{\theta_{k}}{2}-\left(|N|-1-\sigma_{j}^{* *}\right) \frac{\theta_{j}}{2}\right)$. Note that with $t_{j}$ as specified above, at this stage, $j$ 's utility is guaranteed to be no less than her reservation utility, regardless of the queue proposed by $i_{n}$.

Now consider stage 2. Obviously, for any proposed $\sigma, i_{n}$ does not make $j$ an offer $t_{j}$ that

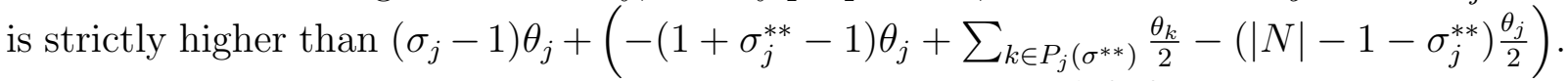
In the meantime, $i_{n}$ would not lower the offer $t_{j}$ for any $j \in N \backslash\left\{i_{n}\right\}$ to be strictly less than $\left(\sigma_{j}-1\right) \theta_{j}+\left(-\left(1+\sigma_{j}^{* *}-1\right) \theta_{j}+\sum_{k \in P_{j}\left(\sigma^{* *}\right)} \frac{\theta_{k}}{2}-\left(|N|-1-\sigma_{j}^{* *}\right) \frac{\theta_{j}}{2}\right)$ since it would lead her proposal to be rejected and then to be served at first. Being served first implies 0 waiting cost for $i_{n}$ at this stage, but there is an incentive for $i_{n}$ to make an acceptable proposal as presented at stage 2 since her payoff from an accepted proposal is

$$
-\left(\sigma_{i_{n}}^{*}-1\right) \theta_{i_{n}}-\sum_{j \in N \backslash\left\{i_{n}\right\}} t_{j}
$$




$$
\begin{aligned}
= & -\left(\sigma_{i_{n}}^{*}-1\right) \theta_{i_{n}}-\sum_{j \in N \backslash\left\{i_{n}\right\}}\left(\sigma_{j}^{*}-1\right) \theta_{j} \\
& -\sum_{j \in N \backslash\left\{i_{n}\right\}}\left(-\left(1+\sigma_{j}^{* *}-1\right) \theta_{j}+\sum_{k \in P_{j}\left(\sigma^{* *}\right)} \frac{\theta_{k}}{2}-\left(|N|-1-\sigma_{j}^{* *}\right) \frac{\theta_{j}}{2}\right) \\
= & -\sum_{i \in N}\left(\sigma_{i}^{*}-1\right) \theta_{i}-\sum_{j \in N \backslash\left\{i_{n}\right\}}\left(-\left(1+\sigma_{j}^{* *}-1\right) \theta_{j}\right)-\sum_{j \in N \backslash\left\{i_{n}\right\}}\left(\sum_{k \in P_{j}\left(\sigma^{* *}\right)} \frac{\theta_{k}}{2}-\left(|N|-1-\sigma_{j}^{* *}\right) \frac{\theta_{j}}{2}\right) \\
= & v^{\max }(N)-v^{\max }\left(N \backslash\left\{i_{n}\right\}\right) \\
\geq & -(|N|-(|N|-1)-1) \theta_{i_{n}} \\
= & 0,
\end{aligned}
$$

where the third equality follows from the definition of $v^{\max }$ and the fact that by definition of an efficient allocation, $\sum_{j \in N \backslash\left\{i_{n}\right\}}\left(\sum_{k \in P_{j}\left(\sigma^{* *}\right)} \frac{\theta_{k}}{2}-\left(|N|-1-\sigma_{j}^{* *}\right) \frac{\theta_{j}}{2}\right)=0$, and the inequality follows from Lemma 3.1.

To verify that the strategies restricted to stage 1 constitute an SPE, note that all net bids equal zero, which follows from the fact that $b_{j}^{i}=\phi_{j}\left(N, v^{\max }\right)-\phi_{j}\left(N \backslash\{i\},\left.v^{\max }\right|_{N \backslash\{i\}}\right)$ where $\left.v^{\max }\right|_{N \backslash\{i\}}(S)=v^{\max }(S)$ for all $S \subseteq N \backslash\{i\}$, and by the balanced contributions property of the Shapley value (Myerson, 1980). ${ }^{5}$ To show that a change in the bids made by player $i$ cannot increase that player's payoff we consider the following two cases: First, if player $i$ changes the vector of her bids so that another player becomes the proposer, this will not change her resulting utility, which would still equal that given by the maximal transfer rule (i.e., her Shapley value of the queueing game $v^{\max }$ ). Second, if she changes the vector of her bids and following it she is still the proposer with positive probability, it must be that her total bid $\left(\sum_{j \in N \backslash\{i\}} b_{j}^{i}\right)$ did not decline, which again means her payoff cannot improve. That is, any deviation of the bidding strategy of player $i$ specified at stage 1 cannot improve her payoff. Hence, no player has an incentive to change her bid, showing that the given strategy profile is an SPE.

Since the proof that any SPE yields the maximal transfer allocation proceeds by a series of claims and goes along the same line as the unicity proof of Theorem 1 in PérezCastrillo and Wettstein (2001), it is provided in the Appendix.

Next we introduce our second game, called the last-served mechanism, which implements the minimal transfer rule. Differently from the first-served mechanism, players compete for the right of being served last in the queue. Alternatively, one can think that

\footnotetext{
${ }^{5}$ A value $\phi$ satisfies the balanced contributions property if $\phi_{i}(N, v)-\phi_{i}\left(N \backslash\{j\},\left.v\right|_{N \backslash\{j\}}\right)=\phi_{j}(N, v)-$ $\phi_{j}\left(N \backslash\{i\},\left.v\right|_{N \backslash\{i\}}\right)$ for all $v \in \Gamma^{N}$ and all $i, j \in N$. In van den Brink and Chun (2012) the implications of this property are investigated in the context of queueing.
} 
players are now demanding compensations for them to be served last, which is in the same light as the ALDB (auctioning the leadership with differentiated bids) mechanism in Moulin (1981). The one with the highest net bid (or lowest net demand if the bids are negative) will be selected as the winner. The winner can decide to take up the last position by herself or sell it to the others. For the latter option, she makes a proposal of a queue and a vector of transfers. If the proposal is rejected, she remains at the last position to be served after all the participating players.

Now we provide a formal description of the last-served mechanism, again based on any set of (active) players $S \subseteq N$.

Last-served mechanism. If there is only one player, $N=\{i\}$, she simply is served last (also first) and the default queue is trivially efficient while no transfer will be made. So this player's utility will be $u_{i}(\sigma, t)=-\left(\sigma_{i}-1\right) \theta_{i}+t_{i}=-0 \theta_{i}+0=0$, which is independent of $\theta_{i}$. When the player set $N=\{1, \ldots, n\}$ consists of two or more players, the mechanism is defined for any set of (active) players $S \subseteq N$, recursively starting with $S=N$.

Stage 1. Bidding for the last position: It is the same as stage 1 of the first-served mechanism, except that the right at the winner's disposal in this mechanism is to be served after, instead of before, the rest of the active players.

Stage 2. Taking up or selling the position: The winner $i_{s}$ decides to either take up the last position by herself or sell it to the others. If taking up the position by herself, player $i_{s}$ will be indeed located at the $s$-th position, i.e., after all other players of $S$ but before all players of $N \backslash S$, and will receive her final utility of $-(s-1) \theta_{i}-$ $\sum_{j \in S \backslash\left\{i_{s}\right\}} b_{j}^{i_{s}}+\sum_{r=s+1}^{n} b_{i_{s}}^{i_{r}}$, where $(s-1) \theta_{i}$ is her waiting cost, $\sum_{j \in S \backslash\left\{i_{s}\right\}} b_{j}^{i_{s}}$ is the sum of the bids she paid at stage 1 , and $\sum_{r=s+1}^{n} b_{i_{s}}^{i_{r}}$ is the sum of the bids she received from previously rejected proposers $i_{s+1}, \ldots, i_{n}$. Moreover, stage 3 will not be evoked, but all players in $S$ other than $i_{s}$ proceed again from stage 1 where the set of active players is $S \backslash\left\{i_{s}\right\}$. If the winner $i_{s}$ decides to sell the position, then $i_{s}$ will make a proposal $\left(\sigma(S),\left(t_{j}\right)_{j \in S}\right)$ consisting of a queue $\sigma(S) \in \Pi(S)$ and a vector of transfers $\left(t_{j}\right)_{j \in S} \in \mathbb{R}^{S}$ such that $\sum_{j \in S} t_{j} \leq 0$. (This offer is additional to the bids paid at stage 1.) The game continues to stage 3 .

Stage 3. Approving or disapproving a proposal: The players in $S$ other than $i_{s}$, sequentially, either accept or reject the proposal. If at least one player rejects the proposal, then it is rejected. Otherwise, it is accepted.

(i) If the proposal is rejected, all players in $S$ other than $i_{s}$ go back to stage 1 where the set of active players is $S \backslash\left\{i_{s}\right\}$. Meanwhile, player $i_{s}$ will be left with her default 
position: the position after the queue of $S \backslash\left\{i_{s}\right\}$ but before $N \backslash S$. Consequently, player $i_{s}$ receives her final utility $-(s-1) \theta_{i}-\sum_{j \in S \backslash\left\{i_{s}\right\}} b_{j}^{i_{s}}+\sum_{r=s+1}^{n} b_{i_{s}}^{i_{r}}$. Note that this is the same utility she would receive if she decided in stage 2 to take up her position.

(ii) If the offer is accepted, we have to distinguish between two cases, $S=N$ and $S \neq$ $N$. If $S=N$, all players agree with the proposer $i_{n}$ on her proposal $\left(\sigma(N),\left(t_{j}\right)_{j \in N}\right)$ and the game ENDS. Each player $j \in N \backslash\left\{i_{n}\right\}$ receives $-\left(\sigma(N)_{j}-1\right) \theta_{j}+b_{j}^{i_{n}}+t_{j}$, and player $i_{n}$ receives $-\left(\sigma(N)_{i_{n}}-1\right) \theta_{i_{n}}-\sum_{j \in N \backslash\left\{i_{n}\right\}} b_{j}^{i_{n}}+t_{i_{n}}$, where $t_{i_{n}}=-\sum_{j \in N \backslash\left\{i_{n}\right\}} t_{j}$. If $S \neq N$, all players in $S$ agree with the proposer $i_{s}$ on her proposal $\left(\sigma(S),\left(t_{j}\right)_{j \in S}\right)$, and the game ENDS. Each player $j \in S \backslash\left\{i_{s}\right\}$ receives $-\left(\sigma(S)_{j}-1\right) \theta_{j}+t_{j}$, and with the bids made by $i_{s}$ and all other previously rejected proposers, player $j$ 's final utility is $-\left(\sigma(S)_{j}-1\right) \theta_{j}+\sum_{k=s}^{n} b_{j}^{i_{k}}+t_{j}$. Player $i_{s}$ receives $-\left(\sigma(S)_{i_{s}}-1\right) \theta_{i_{s}}-\sum_{j \in S \backslash\left\{i_{s}\right\}} b_{j}^{i_{s}}+t_{i_{s}}$, where $t_{i_{s}}=-\sum_{j \in S \backslash\left\{i_{s}\right\}} t_{j}$, and by adding the bids $i_{s}$ received from the previously rejected proposers, the final utility of player $i_{s}$ is $-\left(\sigma(S)_{i_{s}}-1\right) \theta_{i_{s}}-\sum_{j \in S \backslash\left\{i_{s}\right\}} b_{j}^{i_{s}}+$ $\sum_{k=s+1}^{n} b_{i_{s}}^{i_{k}}+t_{i_{s}}$.

We show that for any queueing problem $(N, \theta) \in \mathbb{Q}^{N}$, the last-served mechanism has a unique SPE outcome, which coincides with the payoff vector prescribed by the minimal transfer rule. We begin with the following lemma.

Lemma 3.3 For all $q \in \mathbb{Q}^{N}$, all $i \in N$, and all $S \subseteq N \backslash\{i\}$,

$$
v^{\min }(S \cup\{i\})-v^{\min }(S) \geq-|S| \theta_{i} .
$$

Proof. Let $T \subseteq S$ be the set of players whose unit waiting costs are greater than $\theta_{i}$, and $S \backslash T$ be the set of players whose unit waiting costs are less than or equal to $\theta_{i}$. That is, $\theta_{k} \leq \theta_{i}<\theta_{j}$ for all $j \in T$ and all $k \in S \backslash T$. In an efficient queue $\sigma^{*}$ for $S \cup\{i\}$, player $i$ will be served after players in $T$ but before anyone else in the queue $\sigma^{*}$, so that $\sigma_{i}^{*}=|T|+1{ }^{6}$ That is, $i$ will be served after players in $T$ but before anyone else in the queue $\sigma^{*}$. Thus,

$$
\begin{aligned}
v^{\text {min }}(S \cup\{i\})-v^{\text {min }}(S) & =\left(1-\sigma_{i}^{*}\right) \theta_{i}-\sum_{k \in F_{i}\left(\sigma^{*}\right)} \theta_{k} \\
& =(1-(|T|+1)) \theta_{i}-\sum_{k \in S \backslash T} \theta_{k} \\
& =-|T| \theta_{i}-\sum_{k \in S \backslash T} \theta_{k} \\
& \geq-|T| \theta_{i}-(|S|-|T|) \theta_{i} \\
& =-|S| \theta_{i} .
\end{aligned}
$$

\footnotetext{
${ }^{6}$ Note that the position of $i$ may not be $|T|+1$ if there is a player $j \in S$ with $\theta_{j}=\theta_{i}$. Since the choice of an efficient queue has no effect on $v^{\min }(S \cup\{i\})-v^{\min }(S)$, we can take an efficient queue $\sigma^{*}$ with $\sigma_{i}^{*}=|T|+1$.
} 
Note that the inequality in Lemma 3.3 is strict if $\theta_{i}>\theta_{k}$ for at least one $k \in S \backslash\{i\}$. ${ }^{7}$ This lemma also offers a desirable implication: it pays for player $i$ to join coalition $S$ and form an efficient queue rather than taking up a position after $S$ since the cost for the coalition $S \cup\{i\}$ is not more than the cost of $S$ plus the cost of $i$ when she is served in the position after $S$. Note that the right hand side of the inequality in Lemma 3.3 is the utility of player $i$ when she takes up the position $\sigma_{i}=|S|+1$.

Now we are ready to present our second main result.

Theorem 3.4 For any queueing problem $(N, \theta) \in \mathbb{Q}^{N}$, the last-served mechanism has a unique SPE outcome, which coincides with the payoff vector prescribed by the minimal transfer rule $\varphi^{\min }(N, \theta)$.

Since the proof can be constructed in the same line as that of Theorem 3.2 , it is omitted. ${ }^{8}$

Remark: Since Chun and Hokari (2007) establish a coincidence result between the Shapley value and the nucleolus in queueing problems, together with Theorem 3.2 and 3.4, our games can implement the nucleolus of the corresponding games. ${ }^{9}$

Here we discuss implications of the two mechanisms implementing the maximal and the minimal transfer rules, respectively. In both mechanisms, the players have the same strategies. However, the two mechanisms assign different positions to a winner who decides to take up the position in stage $2,{ }^{10}$ or whose proposal is rejected in stage 3 , of a certain round. In the first-served mechanism this player gets the first position (after the already rejected players), while in the last-served mechanism this player gets the last position (in front of the already rejected players). At first sight, the first-served mechanism seems to be advantageous for the proposer since she takes up the best available position (either in stage 2 or if her proposal is rejected in stage 3). Moreover, the proposer seems to have an incentive of making her proposal be rejected since it gets the best position if her proposal is accepted. On the other hand, in the last-served mechanism, the proposer seems to have an

\footnotetext{
${ }^{7}$ In the proof, if $S \backslash T \neq \emptyset$, then the inequality becomes strict.

${ }^{8}$ The proof can be obtained from the authors on request.

${ }^{9}$ This follows from the fact that in the game $v^{\text {min }}$ the worth of any coalition with two or more players equals the sum of the worths of its two-person subcoalitions, while all singletons have worth zero. Equivalently, only coalitions with cardinalities two can have a nonzero (Harsanyi) dividend. The Shapley value and the nucleolus coincide for such games (see van den Nouweland et al., 1996). For $v^{\text {max }}$, by introducing a zero-normalized game, the coincidence result can be established.

${ }^{10}$ Note that the possibility of taking up the position and leaving the game is not a part of the PérezCastrillo and Wettstein's (2001) mechanism which implements the Shapley value for TU games.
} 
incentive of making her proposal be accepted since it gets the worst position if her proposal is rejected.

The 'value' of being the proposer, and therefore the bids made in stage 1, depends on the position that is at stake for the proposer. As it turns out, in SPE, being the proposer in the first-served mechanism is so attractive that the bids to become the proposer are so high that it eventually leads to a combination of bids (in stage 1) and offers (in stage 2) such that the SPE outcome yields the utility payoffs prescribed by the maximal transfer rule which asks relatively high compensations from the first served player to later served players. On the other hand, in SPE, being the proposer in the last-served mechanism is so unattractive that the bids to become the proposer are so low (in fact, the players want to be paid to become the proposer) that it eventually leads to a combination of bids (in stage 1) and offers (in stage 2) such that the SPE outcome yields the utility payoffs prescribed by the minimal transfer rule which asks relatively low compensations from the first served player to later served players. Requiring a rejected player to be served last leads to an equilibrium outcome corresponding to a rule that yields lower transfers that agents with higher waiting costs have to make to agents with lower waiting costs in compensation for taking up a position in front of the queue.

Our result provides a link between a pessimistic treatment of a proposer and an optimistic treatment of a coalition aligning together for the minimal transfer rule. The minimal transfer rule can be obtained by the last-served mechanism that assigns the last position to the proposer, a pessimistic treatment of a proposer. This rule can also be obtained, as in its original definition of Maniquet (2003), under the optimistic assumption that a coalition is served before the rest of players. Similarly, the first-served mechanism provides a link between an optimistic treatment of a proposer and a pessimistic treatment of a coalition, which in either way leads to the same maximal transfer rule.

The axiomatic foundations of the maximal and the minimal transfer rules indicate that the two rules have desirable properties and are complementary to each other. Next we introduce an average transfer rule that takes the average of these two rules, which can be seen as a compromised choice between the optimistic and the pessimistic perspectives. To construct a strategic mechanism for the average transfer rule, one may combine the two mechanisms by setting up a lottery device in the beginning such that there will be an equal probability to play the first-served and the last-served mechanisms. Each player's expected payoff from playing this mega-game equals the average of the allocations generated by the maximal and the minimal transfer rules. However, this is not a natural implementation mechanism because players' payoffs are generated exogenously and the coincidence only happens in expectation rather than in actual terms. To deal with this difficulty, we offer another mechanism implementing the average transfer rule. 
Hybrid mechanism. The mechanism is the same as the previous two mechanisms, except when the set of active players is $N$.

Stage 1. Bidding for the proposer: It is the same as stage 1 of the previous two mechanisms, except that the winner in this mechanism has an equal chance to be served in the first and the last positions (e.g., by a fair lottery device like flipping a coin), instead of being served for sure either in the first position or in the last position.

Stage 2. Taking the chance or making a proposal: The winner $i_{n}$ decides to either take the chance by herself or make a proposal. If $i_{n}$ takes the chance by herself, a lottery device will generate the actual state for $i_{n}$ to be located first or last. Then, the mechanism proceeds along this realized position for all the other players, with no lottery device anymore. If $i_{n}$ is located first by the lottery device, then the mechanism proceeds as in the first-served mechanism for the other players, and if $i_{n}$ is located last by the lottery device, then the mechanism proceeds as in the last-served mechanism for the other players. On the other hand, if the winner $i_{n}$ decides to make a proposal, then the proposal will consist of a queue $\sigma(N) \in \Pi(N)$ and a vector of transfers $\left(t_{j}\right)_{j \in N} \in \mathbb{R}^{N}$ such that $\sum_{j \in N} t_{j} \leq 0$. The game continues to stage 3 .

Stage 3. Approving or disapproving a proposal: The players in $N$ other than $i_{n}$, sequentially, either accept or reject the proposal. If at least one player rejects the proposal, then it is rejected. Otherwise, it is accepted. If the proposal is rejected, then the lottery device reveals which position, the first or the last, the rejected proposer $i_{n}$ actually takes. For all the other players $N \backslash\left\{i_{n}\right\}$, the mechanism will proceed according to the realized position on $i_{n}$, and there will be no lottery device anymore. That is, if $i_{n}$ takes up the first position, then the other players will play the first-served mechanism. On the contrary, if $i_{n}$ takes up the last position, then the other players will play the last-served mechanism.

To sum up, the only difference takes place when the active set of players is $N$, where a proposer has an equal probability to be served in the first position and the last position. Once the position is revealed, then the mechanism proceeds along this line for the remaining players.

Proposition 3.5 For any queueing problem $(N, \theta) \in \mathbb{Q}^{N}$, the hybrid mechanism has a unique SPE outcome, which coincides with the payoff vector prescribed by the average transfer rule, i.e., $\frac{1}{2}\left(\varphi^{\max }(N, \theta)+\varphi^{\min }(N, \theta)\right)$. 
Proof. Since the proof can be constructed along the same line as in Theorems 3.2 and 3.4, we only provide the sketch. From Theorem 3.2, if the first-served mechanism is played by $N \backslash\{i\}$, then the unique SPE outcome for this subgame is $\varphi^{\max }\left(N \backslash\left\{i_{n}\right\}, \theta_{N \backslash\left\{i_{n}\right\}}\right)$. Similarly, if the last-served mechanism will be played, then the outcome is $\varphi^{\min }\left(N \backslash\left\{i_{n}\right\}, \theta_{N \backslash\left\{i_{n}\right\}}\right)$. Therefore, in SPE, $i_{n}$ will make a proposal such that each player $j \in N \backslash\{j\}$ obtains $\frac{1}{2}\left(\varphi_{j}^{\max }\left(N \backslash\left\{i_{n}\right\}, \theta_{N \backslash\left\{i_{n}\right\}}\right)+\varphi_{j}^{\min }\left(N \backslash\left\{i_{n}\right\}, \theta_{N \backslash\left\{i_{n}\right\}}\right)\right)$, which is player $j$ 's expected payoff if $i_{n}$ is rejected. Due to Lemma 3.1 and Lemma 3.3, one can obviously see that $i_{n}$ has an incentive to make such an acceptable proposal instead of being rejected and receiving the expected payoff $\frac{1}{2}\left(0-(n-1) \theta_{i_{n}}\right)$.

\section{Independence of the ordering of the rejected players}

Now we discuss a robustness property of the two mechanisms implementing the minimal and the maximal transfer rules. Recall that the last-served mechanism requires a rejected proposer to be served after the others. If the proposal of $i_{n}$, as the proposer for the set of active players $N$, is rejected, she will be placed at the last position of the entire queue of $N$, no matter how $N \backslash\left\{i_{n}\right\}$ is arranged. If the proposal of $i_{n-1}$, as the proposer for the set of active players $N \backslash\left\{i_{n}\right\}$ is rejected, $i_{n-1}$ will take up the $(|N|-1)$-th position but before $i_{n}$, and so on. The most recently rejected player will be served after the remaining set of active players, but before the previously rejected players. One may consider an alternative design of the mechanism which assigns to the first rejected proposer the first position of all the rejected players and to the most recently rejected player the last position of all the rejected players. The following proposition confirms that such a mechanism would still implement the minimal transfer rule.

Proposition 4.1 The ordering of the rejected players in the last-served mechanism, so long as they are served after the set of active players $S$, plays no role in implementing the minimal transfer rule of a queueing problem $(N, \theta)$ in $S P E$.

PROOF. Any alternative ordering different from the one specified in the original last-served mechanism would mean that the most recently rejected player cannot do better. That is, $i_{s}$, if rejected, will take the $s$-th position or after. Taking the $s$-th position, the same as in the original last-served mechanism, gives her no incentive to make an unacceptable offer when she was making a proposal. Since a later position can only make her worse off when being rejected, she will make an acceptable offer in SPE. Therefore, even if a rejected player would be placed at the beginning of all the rejected players, she would see that all others will have no incentive to have their offers be rejected and this ordering will give the same result as in the original last-served mechanism. 
Similarly, we can construct an alternative mechanism to implement the maximal transfer rule. In this mechanism, when a proposer is rejected, her final position in the queue of $N$ is pending, up to the moment when the finally rejected proposer is settled. Suppose $S \backslash\left\{i_{s}\right\}$ have made an agreement. The last rejected proposer $i_{s}$ will take up the position immediately before $S \backslash\left\{i_{s}\right\}$ but after $N \backslash S$, whereas all players in $N \backslash S$ can form any ordering.

Proposition 4.2 The ordering of the rejected players in the first-served mechanism, so long as they are served before the set of active players $S$ and the last rejected player takes the position immediately before $S$, plays no role in implementing the maximal transfer rule of a queueing problem $(N, \theta)$ in $S P E$.

PRoOF. A player may wish her successors to become rejected players so that she might be better offer by being placed at a front position. However, in a two-player subgame, the proposer would not make an unacceptable offer. So the previous proposer, for a 3player subgame, can foresee this and realize that she would have to be the finally rejected proposer, if she were to make a proposal being rejected, which implies that she will be placed immediately before the other two players, but not further front. Therefore, she would not make an unacceptable offer, either. Backward induction leads the first proposer to make an acceptable offer, too.

\section{References}

Brink, R. van den and Y. Chun (2012), "Balanced consistency and balanced cost reduction for sequencing problems", Social Choice and Welfare, 38, 519-529.

Chun, Y. (2006a), "A pessimistic approach to the queueing problem" Mathematical Social Sciences, 51, 171-181.

Chun, Y. (2006b), "No-envy in queueing problems" Economic Theory, 29, 151-162.

Chun, Y. (2011), "Consistency and monotonicity in sequencing problems", International Journal of Game Theory, 40, 29-41.

Chun, Y. and T. Hokari (2007), "On the coincidence of the Shapley value and the Nucleolus in queueing problems" Seoul Journal of Economics, 20, 223-237.

Dolan, R. (1978) "Incentive mechanisms for priority queueing problems", Bell Journal of Economics, 9, 421-436. 
Gul, F. (1989) "Bargaining Foundations of Shapely Value", Econometrica, 57, 81-95.

Hart, S., and A. Mas-Colell , (1996), "Bargaining and Value", Econometrica, 64, 357-380.

Ju, Y. (2013) "Efficiency and compromise: a bid-offer counteroffer mechanism with two players", International Journal of Game Theory, 42, 501-520.

Ju, Y. and D. Wettstein (2009) "Implementing Cooperative Solution Concepts: a Generalized Bidding Approach", Economic Theory, 39, 307-330.

Maniquet, F. (2003) "A characterization of the Shapley value in queueing problems", Journal of Economic Theory 109, 90-103.

Mishra, D., and B. Rangarajan (2007) "Cost sharing in a job scheduling problem", Social Choice and Welfare, 29, 369-382.

Mitra, M. (2001) "Mechanism design in queueing problems", Economic Theory, 17, 277305.

Mitra, M. (2002) "Achieving the first best in sequencing problems", Review of Economic Design, 7, 75-91.

Moulin, E. (1981) "Implementing Just and Efficient Decision Making", Journal of Public Economics, 16, 193-213.

Moulin, H. (2007) "On scheduling fees to prevent merging, splitting, and transferring of jobs", Mathematics of Operations Research, 32, 266-283.

Myerson, R.B. (1980) "Conference structures and fair allocation rules", International Journal of Game Theory, 9, 169-182.

Nouweland, A. van den, P. Borm, W. van Golstein Brouwers, R. Groot Bruinderink and S. Tijs (1996) "A game theoretic approach to problems in telecommunication", Management Science, 42, 294-303.

Pérez-Castrillo, D. and D. Wettstein (2001) "Bidding for the Surplus: a Non-Cooperative Approach to the Shapley Value", Journal of Economic Theory 100, 274-294.

Suijs, J. (1996) "On incentive compatibility and budget balancedness in public decision making", Economic Design, 2, 193-209. 


\section{Appendix: Proof of unicity in Theorem 3.2}

The proof that any SPE yields the allocation prescribed by the maximal transfer allocation proceeds by a series of claims.

Claim (1). At stage 3, in any SPE, any player $j \in N \backslash\left\{i_{n}\right\}$ accepts any proposal $(\sigma, t)$ such that $\sigma \in \Pi(N)$, if $t_{j}>\left(\sigma_{j}-1\right) \theta_{j}+\left(-\left(1+\sigma_{j}^{* *}-1\right) \theta_{j}+\sum_{k \in P_{j}\left(\sigma^{* *}\right)} \frac{\theta_{k}}{2}-\left(|N|-1-\sigma_{j}^{* *}\right) \frac{\theta_{j}}{2}\right)$, and rejects it if $t_{j}<\left(\sigma_{j}-1\right) \theta_{j}+\left(-\left(1+\sigma_{j}^{* *}-1\right) \theta_{j}+\sum_{k \in P_{j}\left(\sigma^{* *}\right)} \frac{\theta_{k}}{2}-\left(|N|-1-\sigma_{j}^{* *}\right) \frac{\theta_{j}}{2}\right)$, where $\sigma^{* *} \in \pi\left(N \backslash\left\{i_{n}\right\}, \theta_{N \backslash\left\{i_{n}\right\}}\right)$.

Proof. This claim follows directly from the induction assumption.

Claim (2). (i) If $v^{\max }(N)-v^{\max }\left(N \backslash\left\{i_{n}\right\}\right)>-(|N|-(|N|-1)-1) \theta_{i_{n}}=0$, the only SPE of the game that starts at stage 2 is the following. At stage 2 , player $i_{n}$ chooses the option of selling the position instead of taking it up by herself, and makes a proposal $\left(\sigma^{*}, t\right)$ such that $\sigma^{*} \in \pi(N, \theta)$ and $t_{j}=\left(\sigma_{j}^{*}-1\right) \theta_{j}+\left(-\left(1+\sigma_{j}^{* *}-1\right) \theta_{j}+\sum_{k \in P_{j}\left(\sigma^{* *}\right)} \frac{\theta_{k}}{2}-\left(|N|-1-\sigma_{j}^{* *}\right) \frac{\theta_{j}}{2}\right)$, where $\sigma^{* *} \in \pi\left(N \backslash\left\{i_{n}\right\}, \theta_{N \backslash\left\{i_{n}\right\}}\right)$, to each $j \in N \backslash\left\{i_{n}\right\}$. At stage 3 , each $j \in N \backslash\left\{i_{n}\right\}$ accepts any proposal $(\sigma, t)$ such that $\sigma \in \Pi(N)$, if

$t_{j} \geq\left(\sigma_{j}-1\right) \theta_{j}+\left(-\left(1+\sigma_{j}^{* *}-1\right) \theta_{j}+\sum_{k \in P_{j}\left(\sigma^{* *}\right)} \frac{\theta_{k}}{2}-\left(|N|-1-\sigma_{j}^{* *}\right) \frac{\theta_{j}}{2}\right)$ where $\sigma^{* *} \in$ $\pi\left(N \backslash\left\{i_{n}\right\}, \theta_{N \backslash\left\{i_{n}\right\}}\right)$, and rejects it otherwise.

(ii) If $v^{\max }(N)-v^{\max }\left(N \backslash\left\{i_{n}\right\}\right)=0$, there exist other SPE's in addition to the one described in the main text. In fact, any set of the following strategies also constitutes an SPE: at stage 2 , player $i_{n}$ either takes up the first position by herself or sells the position by making a proposal $(\sigma, t)$ such that $\sigma \in \Pi(N)$ and to some $j \neq i_{n}$,

$t_{j}<\left(\sigma_{j}-1\right) \theta_{j}+\left(-\left(1+\sigma_{j}^{* *}-1\right) \theta_{j}+\sum_{k \in P_{j}\left(\sigma^{* *}\right)} \frac{\theta_{k}}{2}-\left(|N|-1-\sigma_{j}^{* *}\right) \frac{\theta_{j}}{2}\right)$ where $\sigma^{* *} \in$ $\pi\left(N \backslash\left\{i_{n}\right\}, \theta_{N \backslash\left\{i_{n}\right\}}\right)$; and, at stage 3 , player $j$ rejects any proposal $t_{j} \leq\left(\sigma_{j}-1\right) \theta_{j}+$ $\left(-\left(1+\sigma_{j}^{* *}-1\right) \theta_{j}+\sum_{k \in P_{j}\left(\sigma^{* *}\right)} \frac{\theta_{k}}{2}-\left(|N|-1-\sigma_{j}^{* *}\right) \frac{\theta_{j}}{2}\right)$. In any SPE of this subgame, the final payoffs to players $i_{n}$ and $j \neq i_{n}$ are $-\left(\sigma_{i_{n}}^{*}-1\right) \theta_{i_{n}}-\sum_{j \in N \backslash\left\{i_{n}\right\}} b_{j}^{i_{n}}-\sum_{j \in N \backslash\left\{i_{n}\right\}} t_{j}$, and $-\left(\sigma_{j}^{*}-1\right) \theta_{j}+b_{j}^{i_{n}}+t_{j}$, respectively, where

$t_{j}=\left(\sigma_{j}^{*}-1\right) \theta_{j}+\left(-\left(1+\sigma_{j}^{* *}-1\right) \theta_{j}+\sum_{k \in P_{j}\left(\sigma^{* *}\right)} \frac{\theta_{k}}{2}-\left(|N|-1-\sigma_{j}^{* *}\right) \frac{\theta_{j}}{2}\right)$.

Proof. For the case of $v^{\max }(N)-v^{\max }\left(N \backslash\left\{i_{n}\right\}\right)>0$, one can verify the argument by the induction assumption and Lemma 3.1. For the case of $v^{\max }(N)-v^{\max }\left(N \backslash\left\{i_{n}\right\}\right)=0$, one can obviously see that $i_{n}$ would be indifferent between taking up the first position and making an acceptable proposal, with the first option being equivalent to making an unacceptable proposal and then having it be rejected, which would all yield the same payoff to $i_{n}$. Note that in this case $\theta_{i_{n}}>\theta_{j}$ for all $j \in N \backslash\left\{i_{n}\right\}$ due to Lemma 3.1, and by the induction hypothesis, taking up the first position by $i_{n}$ still yields an efficient queue. 
Claim (3). In any SPE, $B^{i}=B^{j}$ for all $i, j \in N$ and hence $B^{i}=0$ for all $i \in N$.

Claim (4). In any SPE, each player's payoff is the same regardless of who is chosen as the winner at stage 1.

Proof. The proofs of claims (3) and (4) are the same as in Pérez-Castrillo and Wettstein (2001).

Claim (5). In any SPE, the final payoff of each player coincides with her payoff prescribed by the maximal transfer rule.

PROOF. Note that if player $i$ is the proposer, her final payoff is $-\left(\sigma_{i}^{*}-1\right) \theta_{i}-\sum_{j \in N \backslash\{i\}} b_{j}^{i}-$ $\sum_{j \in N \backslash\{i\}} t_{j}$, where $t_{j}=\left(\sigma_{j}^{*}-1\right) \theta_{j}+\left(-\left(1+\sigma_{j}^{* *}-1\right) \theta_{j}+\sum_{k \in P_{j}\left(\sigma^{* *}\right)} \frac{\theta_{k}}{2}-\left(|N|-1-\sigma_{j}^{* *}\right) \frac{\theta_{j}}{2}\right)$. And if player $j \neq i$ is the proposer, the final payoff of player $i$ is $-\left(\sigma_{i}^{*}-1\right) \theta_{i}+b_{i}^{j}+$ $\left(\left(\sigma_{i}^{*}-1\right) \theta_{i}+\left(-\left(1+\sigma_{i}^{* *}-1\right) \theta_{i}+\sum_{k \in P_{i}\left(\sigma^{* *}\right)} \frac{\theta_{k}}{2}-\left(|N|-1-\sigma_{i}^{* *}\right) \frac{\theta_{i}}{2}\right)\right)$. Therefore, the sum of payoffs to player $i$ over all possible choices of the proposer is:

$$
\begin{aligned}
& -\left(\sigma_{i}^{*}-1\right) \theta_{i}-\sum_{j \in N \backslash\{i\}} b_{j}^{i} \\
& -\sum_{j \in N \backslash\{i\}}\left(\left(\sigma_{j}^{*}-1\right) \theta_{j}+\left(-\left(1+\sigma_{j}^{* *}-1\right) \theta_{j}+\sum_{k \in P_{j}\left(\sigma^{* *}\right)} \frac{\theta_{k}}{2}-\left(|N|-1-\sigma_{j}^{* *}\right) \frac{\theta_{j}}{2}\right)\right) \\
& +\sum_{j \neq i}\left(-\left(\sigma_{i}^{*}-1\right) \theta_{i}+b_{i}^{j}\right) \\
& +\sum_{j \neq i}\left(\left(\sigma_{i}^{*}-1\right) \theta_{i}+\left(-\left(1+\sigma_{i}^{* *}-1\right) \theta_{i}+\sum_{k \in P_{i}\left(\sigma^{* *}\right)} \frac{\theta_{k}}{2}-\left(|N|-1-\sigma_{i}^{* *}\right) \frac{\theta_{i}}{2}\right)\right) \\
& =-n\left(\sigma_{i}^{*}-1\right) \theta_{i}-\sum_{j \in N \backslash\{i\}}\left(\left(\sigma_{j}^{*}-1\right) \theta_{j}+\left(-\left(1+\sigma_{j}^{* *}-1\right) \theta_{j}+\sum_{k \in P_{j}\left(\sigma^{* *}\right)} \frac{\theta_{k}}{2}-\left(|N|-1-\sigma_{j}^{* *}\right) \frac{\theta_{j}}{2}\right)\right. \\
& +(n-1)\left(\sigma_{i}^{*}-1\right) \theta_{i}+\sum_{j \neq i}\left(-\left(1+\sigma_{i}^{* *}-1\right) \theta_{i}+\sum_{k \in P_{i}\left(\sigma^{* *}\right)} \frac{\theta_{k}}{2}-\left(|N|-1-\sigma_{i}^{* *}\right) \frac{\theta_{i}}{2}\right) \\
& =-\left(\sigma_{i}^{*}-1\right) \theta_{i}-\sum_{j \in N \backslash\{i\}}\left(\sigma_{j}^{*}-1\right) \theta_{j}-\sum_{j \in N \backslash\{i\}}\left(-\sigma_{j}^{* *} \theta_{j}\right) \\
& +\sum_{j \in N \backslash\{i\}}\left(-\sigma_{i}^{* *} \theta_{i}+\sum_{k \in P_{i}\left(\sigma^{* *}\right)} \frac{\theta_{k}}{2}-\left(|N|-1-\sigma_{i}^{* *}\right) \frac{\theta_{i}}{2}\right) \\
& =-\left(\sigma_{i}^{*}-1\right) \theta_{i}+\sum_{k \in P_{i}\left(\sigma^{*}\right)} \theta_{k}+\sum_{j \in N \backslash\{i\}}\left(-(|N|-1) \frac{\theta_{i}}{2}-\sigma_{i}^{* *} \frac{\theta_{i}}{2}+\sum_{k \in P_{i}\left(\sigma^{* *}\right)} \frac{\theta_{k}}{2}\right)
\end{aligned}
$$




$$
\begin{array}{ll}
= & -\left(\sigma_{i}^{*}-1\right) \theta_{i}+\sum_{k \in P_{i}\left(\sigma^{*}\right)} \theta_{k}-\sum_{j \in N \backslash\{i\}} \frac{\sigma_{i}^{* *} \theta_{i}}{2}-\frac{(n-1)^{2}}{2} \theta_{i}+\sum_{j \in N \backslash\{i\}} \sum_{k \in P_{i}\left(\sigma^{* *}\right)} \frac{\theta_{k}}{2} \\
= & -\left(\sigma_{i}^{*}-1\right) \theta_{i}+\sum_{k \in P_{i}\left(\sigma^{*}\right)} \theta_{k}-\frac{1}{2}\left(\left|P_{i}\left(\sigma^{*}\right)\right|\left(\sigma^{*}-1\right) \theta_{i}+\left|F_{i}\left(\sigma^{*}\right)\right| \sigma^{*} \theta_{i}\right)-\frac{(n-1)^{2}}{2} \theta_{i} \\
& +\left|F_{i}\left(\sigma^{*}\right)\right| \sum_{k \in P_{i}\left(\sigma^{*}\right)} \frac{\theta_{k}}{2}+\left(\left|P_{i}\left(\sigma^{*}\right)\right|-1\right) \sum_{k \in P_{i}\left(\sigma^{*}\right)} \frac{\theta_{k}}{2} \\
= & -\left(\sigma_{i}^{*}-1\right) \theta_{i}-\frac{1}{2}\left((n-1) \sigma^{*} \theta_{i}-\left|P_{i}\left(\sigma^{*}\right)\right| \theta_{i}\right)-\frac{(n-1)^{2}}{2} \theta_{i}+\sum_{k \in P_{i}\left(\sigma^{*}\right)} \frac{n \theta_{k}}{2} \\
= & -\left(\sigma_{i}^{*}-1\right) \theta_{i}-\frac{1}{2}\left((n-1) \sigma^{*} \theta_{i}-\left(\sigma_{i}^{*}-1\right) \theta_{i}\right)-\frac{(n-1)^{2}}{2} \theta_{i}+\sum_{k \in P_{i}\left(\sigma^{*}\right)} \frac{n \theta_{k}}{2} \\
= & -\frac{n}{2}\left(\sigma_{i}^{*}-2+n\right) \theta_{i}+\sum_{k \in P_{i}\left(\sigma^{*}\right)} \frac{n \theta_{k}}{2} \\
= & n\left(-\left(\sigma_{i}^{*}-1\right) \theta_{i}-\left(n-\sigma_{i}^{*}\right) \frac{\theta_{i}}{2}+\sum_{k \in P_{i}\left(\sigma^{*}\right)} \frac{\theta_{k}}{2}\right) \\
= & n \varphi_{i}^{\max }(N, \theta) .
\end{array}
$$

Together with Claim (4), we can conclude that in any SPE, for any player $i \in N$, her final payoff is $\varphi_{i}^{\max }(N, \theta)$. 


\section{Annex (not for publication): Proof of Theorem 3.4}

Consider a queueing problem $(N, \theta) \in \mathbb{Q}^{N}$. The proof proceeds by induction on the number of players $n$. The induction assumption is that whenever the mechanism is used by $n$ players with a given vector of unit waiting costs, it implements the minimal transfer rule to this queueing problem. It is easy to see that the theorem holds for $n=1$. We assume that it holds for all $m \leq n-1$ and show that it is satisfied for $n$.

First we show that the minimal transfer rule is an SPE outcome. We explicitly construct an SPE that yields the minimal transfer allocation as the SPE outcome. Consider the following strategies, which the players would follow in any (sub)game they participate in (we describe it for the whole set of players, $N$, but similar strategies are followed by any player in $S \subseteq N$ that is called upon to play the game, with $S$ replacing $N$ ):

At stage 1, each player $i \in N$ announces

$$
b_{j}^{i}=\left(-\left(\sigma_{j}^{*}-1\right) \theta_{j}+\left(\sigma_{j}^{*}-1\right) \frac{\theta_{j}}{2}-\sum_{k \in F_{j}\left(\sigma^{*}\right)} \frac{\theta_{k}}{2}\right)-\left(-\left(\sigma_{j}^{* *}-1\right) \theta_{j}+\left(\sigma_{j}^{* *}-1\right) \frac{\theta_{j}}{2}-\sum_{k \in F_{j}\left(\sigma^{* *}\right)} \frac{\theta_{k}}{2}\right)
$$

where $\sigma^{*} \in \pi(N, \theta)$ and $\sigma^{* *} \in \pi\left(N \backslash\left\{i_{n}\right\}, \theta_{N \backslash\left\{i_{n}\right\}}\right)$ for all $j \in N \backslash\{i\}$.

At stage 2 , the proposer, player $i_{n}$, adopts the option of selling the position instead of taking it up by herself, and makes a proposal $\left(\sigma^{*}, t\right)$ such that $\sigma^{*} \in \pi(N, \theta)$ and $j \in N \backslash\left\{i_{n}\right\}$, $t_{j}=\left(\sigma_{j}^{*}-1\right) \theta_{j}+\left(-\left(\sigma_{j}^{* *}-1\right) \theta_{j}+\left(\sigma_{j}^{* *}-1\right) \frac{\theta_{j}}{2}-\sum_{k \in F_{j}\left(\sigma^{* *}\right)} \frac{\theta_{k}}{2}\right)$, where $\sigma^{* *} \in \pi\left(N \backslash\left\{i_{n}\right\}, \theta_{N \backslash\left\{i_{n}\right\}}\right)$.

At stage 3, any player $j \in N \backslash\left\{i_{n}\right\}$ accepts any proposal $(\sigma, t)$ if $\sigma \in \Pi(N)$ and $t_{j} \geq$ $\left(\sigma_{j}-1\right) \theta_{j}+\left(-\left(\sigma_{j}^{* *}-1\right) \theta_{j}+\left(\sigma_{j}^{* *}-1\right) \frac{\theta_{j}}{2}-\sum_{k \in F_{j}\left(\sigma^{* *}\right)} \frac{\theta_{k}}{2}\right)$ where $\sigma^{* *} \in \pi\left(N \backslash\left\{i_{n}\right\}, \theta_{N \backslash\left\{i_{n}\right\}}\right)$, and rejects it otherwise.

To verify the above strategies indeed constitute an SPE and yield the minimal transfer rule, we first look at stage 3 . Suppose the proposal of $i_{n}$ is rejected. From the induction hypothesis, we know that for the remaining players $N \backslash\left\{i_{n}\right\}$, a corresponding efficient queue will be formed and the resulting utility to every player $j \in N \backslash\left\{i_{n}\right\}$ is $-\left(\sigma_{j}^{* *}-1\right) \theta_{j}+$ $\left(\sigma_{j}^{* *}-1\right) \frac{\theta_{j}}{2}-\sum_{k \in F_{j}\left(\sigma^{* *}\right)} \frac{\theta_{k}}{2}$, where $\sigma^{* *} \in \pi\left(N \backslash\left\{i_{n}\right\}, \theta_{N \backslash\left\{i_{n}\right\}}\right)$, which is the reservation utility for $j$ when she considers any proposal made by $i_{n}$. If $j$ accepts the proposal made by $i_{n}$, she receives the utility $-\left(\sigma_{j}-1\right) \theta_{j}+t_{j}$. Apparently, only when this utility is no less than her reservation utility, she will accept the proposal, which gives rise to $t_{j} \geq$ $\left(\sigma_{j}-1\right) \theta_{j}+\left(-\left(\sigma_{j}^{* *}-1\right) \theta_{j}+\left(\sigma_{j}^{* *}-1\right) \frac{\theta_{j}}{2}-\sum_{k \in F_{j}\left(\sigma^{* *}\right)} \frac{\theta_{k}}{2}\right)$. Note that with $t_{j}$ as specified above, at this stage, player $j$ 's utility is guaranteed to be no less than her reservation utility, regardless of the queue proposed by $i_{n}$.

Now consider stage 2. Obviously, for any proposed $\sigma, i_{n}$ does not make an offer $t_{j}$ that is strictly higher than $\left(\sigma_{j}-1\right) \theta_{j}+\left(-\left(\sigma_{j}^{* *}-1\right) \theta_{j}+\left(\sigma_{j}^{* *}-1\right) \frac{\theta_{j}}{2}-\sum_{k \in F_{j}\left(\sigma^{* *}\right)} \frac{\theta_{k}}{2}\right)$ 
for any $j \in N \backslash\left\{i_{n}\right\}$. In the meantime, $i_{n}$ would not lower $t_{j}$ to be strictly less than $\left(\sigma_{j}-1\right) \theta_{j}+\left(-\left(\sigma_{j}^{* *}-1\right) \theta_{j}+\left(\sigma_{j}^{* *}-1\right) \frac{\theta_{j}}{2}-\sum_{k \in F_{j}\left(\sigma^{* *}\right)} \frac{\theta_{k}}{2}\right)$ for any $j \in N \backslash\left\{i_{n}\right\}$ since it would lead her proposal to be rejected and served at last with the waiting cost of $-(|N|-1) \theta_{i_{n}}$ at this stage. We show that $i_{n}$ does have an incentive to make such an acceptable proposal. By making this proposal at stage 2 , player $i_{n}$ receives

$$
\begin{aligned}
& -\left(\sigma_{i_{n}}-1\right) \theta_{i_{n}}-\sum_{j \in N \backslash\left\{i_{n}\right\}} t_{j} \\
= & -\left(\sigma_{i_{n}}-1\right) \theta_{i_{n}}-\sum_{j \in N \backslash\left\{i_{n}\right\}}\left(\left(\sigma_{j}-1\right) \theta_{j}+\left(-\left(\sigma_{j}^{* *}-1\right) \theta_{j}+\left(\sigma_{j}^{* *}-1\right) \frac{\theta_{j}}{2}-\sum_{k \in F_{j}\left(\sigma^{* *}\right)} \frac{\theta_{k}}{2}\right)\right) \\
= & -\sum_{i \in N}\left(\sigma_{i}-1\right) \theta_{i}-\sum_{j \in N \backslash\left\{i_{n}\right\}}\left(-\left(\sigma_{j}^{* *}-1\right) \theta_{j}\right)-\sum_{j \in N \backslash\left\{i_{n}\right\}}\left(\left(\sigma_{j}^{* *}-1\right) \frac{\theta_{j}}{2}-\sum_{k \in F_{j}\left(\sigma^{* *}\right)} \frac{\theta_{k}}{2}\right) \\
= & -\sum_{i \in N}\left(\sigma_{i}-1\right) \theta_{i}-v^{\min }\left(N \backslash\left\{i_{n}\right\}\right),
\end{aligned}
$$

where the last equality follows from the definition of $v^{\min }$ and the fact that $\sum_{j \in N \backslash\left\{i_{n}\right\}}\left(\left(\sigma_{j}^{* *}-1\right) \frac{\theta_{j}}{2}-\sum_{k \in F_{j}\left(\sigma^{* *}\right)} \frac{\theta_{k}}{2}\right)=0$. Moreover, in order for the proposer $i_{n}$ to maximize what she can achieve, it is obvious that she chooses $\sigma^{*} \in \pi(N, \theta)$ because $-\sum_{i \in N}\left(\sigma_{i}^{*}-1\right) \theta_{i} \geq-\sum_{i \in N}\left(\sigma_{i}-1\right) \theta_{i}$ for all $\sigma \in \Pi(N, \theta) \backslash \pi(N, \theta)$. Hence, $i_{n}$ will receive $v^{\min }(N)-v^{\min }\left(N \backslash\left\{i_{n}\right\}\right) \geq-(|N|-1) \theta_{i_{n}}$, where the inequality follows from Lemma 3.3.

To verify the strategies restricted to stage 1 constitute an SPE, note that all net bids equal zero, which follows from the fact that $b_{j}^{i}=\phi_{j}\left(N, v^{\min }\right)-\phi_{j}\left(N \backslash\{i\},\left.v^{\min }\right|_{N \backslash\{i\}}\right)$ where $\left.v^{m i n}\right|_{N \backslash\{i\}}(S)=v^{\min }(S)$ for all $S \subseteq N \backslash\{i\}$, and by the balanced contributions property of the Shapley value (Myerson, 1980). To show that a change in the bids made by player $i$ cannot increase that player's payoff we consider the following two cases: First, if player $i$ may change the vector of her bids so that another player becomes the proposer, this will not change her resulting utility, which would still equal that given by the minimal transfer rule (i.e., her Shapley value of the queueing game $v^{\text {min }}$ ). Second, if she changes the vector of her bids and following it she is still the proposer with positive probability, it must be that her total bid $\left(\sum_{j \in N \backslash\{i\}} b_{j}^{i}\right)$ did not decline, which again means her payoff cannot improve. That is, any deviation of the bidding strategy of player $i$ specified at stage 1 cannot improve her payoff. Hence, no player has an incentive to change her bid, showing that the given strategy profile is an SPE.

The proof that any SPE yields the allocation prescribed by the minimal transfer rule proceeds by a series of claims, similar to the unicity proof of Theorem 1 in Pérez-Castrillo and Wettstein (2001).

Claim (1). At stage 3, in any SPE, any player $j \in N \backslash\left\{i_{n}\right\}$ accepts any proposal $(\sigma, t)$ 
such that $\sigma \in \Pi(N)$, if $t_{j}>\left(\sigma_{j}-1\right) \theta_{j}+\left(-\left(\sigma_{j}^{* *}-1\right) \theta_{j}+\left(\sigma_{j}^{* *}-1\right) \frac{\theta_{j}}{2}-\sum_{k \in F_{j}\left(\sigma^{* *}\right)} \frac{\theta_{k}}{2}\right)$, and rejects it if $t_{j}<\left(\sigma_{j}-1\right) \theta_{j}+\left(-\left(\sigma_{j}^{* *}-1\right) \theta_{j}+\left(\sigma_{j}^{* *}-1\right) \frac{\theta_{j}}{2}-\sum_{k \in F_{j}\left(\sigma^{* *}\right)} \frac{\theta_{k}}{2}\right)$, where $\sigma^{* *} \in \pi\left(N \backslash\left\{i_{n}\right\}, \theta_{N \backslash\left\{i_{n}\right\}}\right)$.

Proof. This claims follows directly from the induction assumption.

Claim (2). (i) If $v^{\min }(N)-v^{\min }\left(N \backslash\left\{i_{n}\right\}\right)>-(|N|-1) \theta_{i_{n}}$, the only SPE of the game that starts at stage 2 is the following. At stage 2, player $i_{n}$ adopts the option of selling the position instead of taking it up by herself, and makes a proposal $\left(\sigma^{*}, t\right)$ such that $\sigma^{*} \in$ $\pi(N, \theta)$ and $t_{j}=\left(\sigma_{j}^{*}-1\right) \theta_{j}+\left(-\left(\sigma_{j}^{* *}-1\right) \theta_{j}+\left(\sigma_{j}^{* *}-1\right) \frac{\theta_{j}}{2}-\sum_{k \in F_{j}\left(\sigma^{* *}\right)} \frac{\theta_{k}}{2}\right)$, where $\sigma^{* *} \in$ $\pi\left(N \backslash\left\{i_{n}\right\}, \theta_{N \backslash\left\{i_{n}\right\}}\right)$, to each $j \in N \backslash\left\{i_{n}\right\}$. At stage 3 , each $j \in N \backslash\left\{i_{n}\right\}$ accepts any proposal $(\sigma, t)$ such that $\sigma \in \Pi(N)$, if $t_{j} \geq\left(\sigma_{j}-1\right) \theta_{j}+\left(-\left(\sigma_{j}^{* *}-1\right) \theta_{j}+\left(\sigma_{j}^{* *}-1\right) \frac{\theta_{j}}{2}-\sum_{k \in F_{j}\left(\sigma^{* *}\right)} \frac{\theta_{k}}{2}\right)$, where $\sigma^{* *} \in \pi\left(N \backslash\left\{i_{n}\right\}, \theta_{N \backslash\left\{i_{n}\right\}}\right)$, and rejects it otherwise.

(ii) If $v^{\min }(N)-v^{\min }\left(N \backslash\left\{i_{n}\right\}\right)=-(|N|-1) \theta_{i_{n}}$, there exist other SPE's in addition to the above one. In fact, any set of the following strategies also constitutes an SPE: at stage 2, player $i_{n}$ either takes up the last position by herself or sells the position by making a proposal $(\sigma, t)$ such that $\sigma \in \Pi(N)$ and to some $j \neq i_{n}, t_{j}<\left(\sigma_{j}-1\right) \theta_{j}+$ $\left(-\left(\sigma_{j}^{* *}-1\right) \theta_{j}+\left(\sigma_{j}^{* *}-1\right) \frac{\theta_{j}}{2}-\sum_{k \in F_{j}\left(\sigma^{* *}\right)} \frac{\theta_{k}}{2}\right)$ where $\sigma^{* *} \in \pi\left(N \backslash\left\{i_{n}\right\}, \theta_{N \backslash\left\{i_{n}\right\}}\right)$; and at stage 3 , the player $j$ rejects any proposal $t_{j} \leq\left(\sigma_{j}-1\right) \theta_{j}+\left(-\left(\sigma_{j}^{* *}-1\right) \theta_{j}+\left(\sigma_{j}^{* *}-1\right) \frac{\theta_{j}}{2}-\sum_{k \in F_{j}\left(\sigma^{* *}\right)} \frac{\theta_{k}}{2}\right)$. In any SPE of this subgame, the final payoffs to players $i_{n}$ and $j \neq i_{n}$ are $-\left(\sigma_{i_{n}}^{*}-\right.$ 1) $\theta_{i_{n}}-\sum_{j \in N \backslash\left\{i_{n}\right\}} b_{j}^{i_{n}}-\sum_{j \in N \backslash\left\{i_{n}\right\}} t_{j}$, and $-\left(\sigma_{j}^{*}-1\right) \theta_{j}+b_{j}^{i_{n}}+t_{j}$, respectively, where $t_{j}=$ $\left(\sigma_{j}^{*}-1\right) \theta_{j}+\left(-\left(\sigma_{j}^{* *}-1\right) \theta_{j}+\left(\sigma_{j}^{* *}-1\right) \frac{\theta_{j}}{2}-\sum_{k \in F_{j}\left(\sigma^{* *}\right)} \frac{\theta_{k}}{2}\right)$.

Proof. For the case of $v^{\min }(N)-v^{\min }\left(N \backslash\left\{i_{n}\right\}\right)>-(|N|-1) \theta_{i_{n}}$, one can verify the argument by the induction assumption and Lemma 3.3. For the case of $v^{\min }(N)-$ $v^{\min }\left(N \backslash\left\{i_{n}\right\}\right)=-(|N|-1) \theta_{i_{n}}$, one can obviously see that $i_{n}$ would be indifferent between taking up the last position and making an acceptable proposal, with the first option being equivalent to making an unacceptable proposal and then having it be rejected, which would all yield the same payoff to $i_{n}$.

Claim (3). In any SPE, $B^{i}=B^{j}$ for all $i, j \in N$ and hence $B^{i}=0$ for all $i \in N$.

Claim (4). In any SPE, each player's payoff is the same regardless of who is chosen as the winner at stage 1 .

Proof. The proofs of claims (3) and (4) are the same as in Pérez-Castrillo and Wettstein (2001). 
Claim (5). In any SPE, the final payoff of each player coincides with her payoff prescribed by the minimal transfer rule.

Proof. Note that if player $i$ is the proposer, her final payoff is $-\left(\sigma_{i}^{*}-1\right) \theta_{i}-\sum_{j \in N \backslash\{i\}} b_{j}^{i}-$ $\sum_{j \in N \backslash\{i\}} t_{j}$, where $t_{j}=\left(\sigma_{j}^{*}-1\right) \theta_{j}+\left(-\left(\sigma_{j}^{* *}-1\right) \theta_{j}+\left(\sigma_{j}^{* *}-1\right) \frac{\theta_{j}}{2}-\sum_{k \in F_{j}\left(\sigma^{* *}\right)} \frac{\theta_{k}}{2}\right)$. And if player $j \neq i$ is the proposer, the final payoff of player $i$ is $-\left(\sigma_{i}^{*}-1\right) \theta_{i}+b_{i}^{j}+\left(\left(\sigma_{i}^{*}-1\right) \theta_{i}+\left(-\left(\sigma_{i}^{* *}-1\right) \theta_{i}+\left(\sigma_{i}^{* *}-1\right) \frac{\theta_{i}}{2}-\sum_{k \in F_{i}\left(\sigma^{* *}\right)} \frac{\theta_{k}}{2}\right)\right)$. Therefore, the sum of payoffs to player $i$ over all possible choices of the proposer is:

$$
\begin{aligned}
& -\left(\sigma_{i}^{*}-1\right) \theta_{i}-\sum_{j \in N \backslash\{i\}} b_{j}^{i} \\
& -\sum_{j \in N \backslash\{i\}}\left(\left(\sigma_{j}^{*}-1\right) \theta_{j}+\left(-\left(\sigma_{j}^{* *}-1\right) \theta_{j}+\left(\sigma_{j}^{* *}-1\right) \frac{\theta_{j}}{2}-\sum_{k \in F_{j}\left(\sigma^{* *}\right)} \frac{\theta_{k}}{2}\right)\right) \\
& +\sum_{j \neq i}\left(-\left(\sigma_{i}^{*}-1\right) \theta_{i}+b_{i}^{j}\right) \\
& +\sum_{j \neq i}\left(\left(\sigma_{i}^{*}-1\right) \theta_{i}+\left(-\left(\sigma_{i}^{* *}-1\right) \theta_{i}+\left(\sigma_{i}^{* *}-1\right) \frac{\theta_{i}}{2}-\sum_{k \in F_{i}\left(\sigma^{* *}\right)} \frac{\theta_{k}}{2}\right)\right) \\
& =-n\left(\sigma_{i}^{*}-1\right) \theta_{i}-\sum_{j \in N \backslash\{i\}}\left(\left(\sigma_{j}^{*}-1\right) \theta_{j}+\left(-\left(\sigma_{j}^{* *}-1\right) \theta_{j}+\left(\sigma_{j}^{* *}-1\right) \frac{\theta_{j}}{2}-\sum_{k \in F_{j}\left(\sigma^{* *}\right)} \frac{\theta_{k}}{2}\right)\right) \\
& +(n-1)\left(\sigma_{i}^{*}-1\right) \theta_{i}+\sum_{j \neq i}\left(-\left(\sigma_{i}^{* *}-1\right) \theta_{i}+\left(\sigma_{i}^{* *}-1\right) \frac{\theta_{i}}{2}-\sum_{k \in F_{i}\left(\sigma^{* *}\right)} \frac{\theta_{k}}{2}\right) \\
& =\quad-\left(\sigma_{i}^{*}-1\right) \theta_{i}-\sum_{j \in N \backslash\{i\}}\left(\sigma_{j}^{*}-1\right) \theta_{j}-\sum_{j \in N \backslash\{i\}}\left(-\left(\sigma_{j}^{* *}-1\right) \theta_{j}\right) \\
& +\sum_{j \in N \backslash\{i\}}\left(-\left(\sigma_{i}^{* *}-1\right) \theta_{i}+\left(\sigma_{i}^{* *}-1\right) \frac{\theta_{i}}{2}-\sum_{k \in F_{i}\left(\sigma^{* *}\right)} \frac{\theta_{k}}{2}\right) \\
& =-\left(\sigma_{i}^{*}-1\right) \theta_{i}-\sum_{k \in F_{i}\left(\sigma^{*}\right)} \theta_{k}+\sum_{j \in N \backslash\{i\}}\left(-\left(\sigma_{i}^{* *}-1\right) \frac{\theta_{i}}{2}-\sum_{k \in F_{i}\left(\sigma^{* *}\right)} \frac{\theta_{k}}{2}\right) \\
& =\quad-\sigma_{i}^{*} \theta_{i}+\theta_{i}-\sum_{k \in F_{i}\left(\sigma^{*}\right)} \theta_{k}-\sum_{j \in N \backslash\{i\}} \frac{\sigma_{i}^{* *} \theta_{i}}{2}+(n-1) \frac{\theta_{i}}{2}-\sum_{j \in N \backslash\{i\}} \sum_{k \in F_{i}\left(\sigma^{* *}\right)} \frac{\theta_{k}}{2} \\
& =\quad-\sigma_{i}^{*} \theta_{i}-\sum_{k \in F_{i}\left(\sigma^{*}\right)} \theta_{k}-\sum_{j \in N \backslash\{i\}} \frac{\sigma_{i}^{* *} \theta_{i}}{2}+(n+1) \frac{\theta_{i}}{2}-\sum_{j \in N \backslash\{i\}} \sum_{k \in F_{i}\left(\sigma^{* *}\right)} \frac{\theta_{k}}{2} \\
& =\quad-\sigma_{i}^{*} \theta_{i}-\sum_{k \in F_{i}\left(\sigma^{*}\right)} \theta_{k}-\frac{1}{2}\left(\left|F_{i}\left(\sigma^{*}\right)\right| \sigma_{i}^{*} \theta_{i}+\left|P_{i}\left(\sigma^{*}\right)\right|\left(\sigma_{i}^{*}-1\right) \theta_{i}\right)+(n+1) \frac{\theta_{i}}{2}-\sum_{j \in N \backslash\{i\}} \sum_{k \in F_{i}\left(\sigma^{* *}\right)} \frac{\theta_{k}}{2}
\end{aligned}
$$




$$
\begin{aligned}
& =\quad-\frac{n+1}{2}\left(\sigma_{i}^{*}-1\right) \theta_{i}+\frac{1}{2}\left|P_{i}\left(\sigma^{*}\right)\right| \theta_{i}-\sum_{k \in F_{i}\left(\sigma^{*}\right)} \theta_{k}-\frac{1}{2}\left(\left|P_{i}\left(\sigma^{*}\right)\right|+\left|F_{i}\left(\sigma^{*}\right)\right|-1\right) \sum_{k \in F_{i}\left(\sigma^{*}\right)} \theta_{k} \\
& =\quad-\frac{n+1}{2}\left(\sigma_{i}^{*}-1\right) \theta_{i}+\frac{1}{2}\left(\sigma_{i}^{*}-1\right) \theta_{i}-\frac{n}{2} \sum_{k \in F_{i}\left(\sigma^{*}\right)} \theta_{k} \\
& =\quad-\frac{n}{2}\left(\sigma_{i}^{*}-1\right) \theta_{i}-\sum_{k \in F_{i}\left(\sigma^{*}\right)} \frac{n \theta_{k}}{2} \\
& =n\left(-\left(\sigma_{i}^{*}-1\right) \theta_{i}+\left(\sigma_{i}^{*}-1\right) \frac{\theta_{i}}{2}-\sum_{k \in F_{i}\left(\sigma^{*}\right)} \frac{\theta_{k}}{2}\right) \\
& =n \varphi_{i}^{\min }(N, \theta) .
\end{aligned}
$$

Together with Claim (4), we can conclude that in any SPE, for any player $i \in N$, her final payoff is $\varphi_{i}^{\min }(N, \theta)$. 